\title{
Homobenzotetramisole: An EFfective CATAlyst for Kinetic Resolution of ARYL-
} CYCLOALKANOLS

Vladimir B. Birman* and Ximin Li

A contribution from the Department of Chemistry, Washington University, Campus Box 1134, One Brookings Drive, Saint Louis, Missouri 63130

SUPPORTING INFORMATION: Experimental procedures (12 pages), ${ }^{1} \mathrm{H}$ NMR and ${ }^{13} \mathrm{C}$ NMR spectra (28 pages).

\section{EXPERIMENTAL PART.}

1. General. All reagents were obtained commercially and used as received unless otherwise specified. Catalysts $\mathbf{1}^{1 \mathrm{a}}, \mathbf{2}^{1 \mathrm{~b}}$ and $\mathbf{4}^{\mathrm{lc}}$ were prepared as previously described. Substrates $\mathbf{2 8},{ }^{\mathbf{1 3}} \mathbf{2 9},{ }^{\mathbf{1 4}}$ $30,{ }^{15} 31,{ }^{16}$ and $32^{17}$ were prepared according to reported procedures. The syntheses of previously unknown substrates 26 and 27 were performed according to a known general protocol ${ }^{18}$ and are unoptimized. A description of other general experimental details is given in Ref. $1 \mathrm{~b}$.

\section{Preparation of (S)-HTM}

\section{(S)-3-(2-Thiazolinylamino)-3-phenylpropanol-1 (20)}

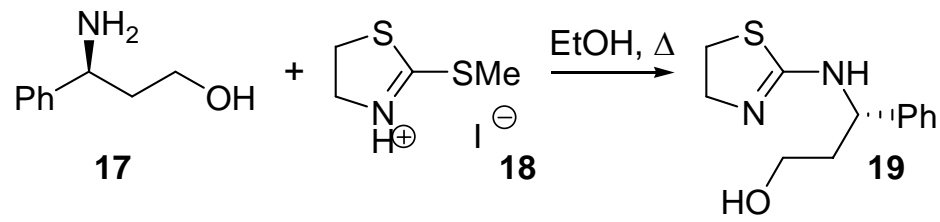

A solution of (S)-3-amino-3-phenylpropanol $\mathbf{1 7}^{6,19}(0.218 \mathrm{~g}, 1.44 \mathrm{mmol})$ and 4, 5-dihydro-2methylthio-thiazole hydriodide $\mathbf{1 8}^{7}(0.414 \mathrm{~g}, 1.59 \mathrm{mmol})$ in $0.5 \mathrm{ml}$ of $95 \% \mathrm{EtOH}$ was refluxed for $15 \mathrm{~h}$. The reaction mixture was basified with $20 \% \mathrm{NaOH}$ and extracted with $\mathrm{CH}_{2} \mathrm{Cl}_{2}$ three times. The organic phase was dried over $\mathrm{Na}_{2} \mathrm{SO}_{4}$. After flash chromatography (10\% isopropanol,

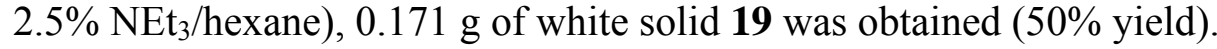

${ }^{1} \mathbf{H}$ NMR $\left(300 \mathrm{MHz}, \mathrm{CDCl}_{3}\right): \delta 7.38-7.25(\mathrm{~m}, 5 \mathrm{H}), 5.07\left(\mathrm{dd}, J_{1}=3.4 \mathrm{~Hz}, J_{2}=11.0 \mathrm{~Hz}, 1 \mathrm{H}\right), 5.0$ (br, $1 \mathrm{H}), 4.06-3.86(\mathrm{~m}, 2 \mathrm{H}), 3.72-3.67(\mathrm{~m}, 1 \mathrm{H}), 3.39-3.30(\mathrm{~m}, 2 \mathrm{H}), 2.12-2.02(\mathrm{~m}, 1 \mathrm{H}), 1.87-1.78$

\footnotetext{
${ }^{13}$ Shepherd T. A.; Aikins J. A.; Bleakman D.; Cantrell B. E.; Rearick J. P.; Simon R. L.; Smith E. C. R.; Stephenson G. A.; Zimmerman D. M.; Mandelzys A.; Jarvie K. R., Ho K.; Deverill M.; Kamboj R. K. J. Med. Chem. 2002, 45, 2101.

${ }^{14}$ Yin J.; Huffman M. A.; Conrad K. M.; Armstrong J. D. J. Org. Chem. 2006, 71, 840.

${ }^{15}$ Iwasaki F.; Maki T.; Onomura O.; Nakashima W.; Matsumura Y. J. Org. Chem., 2000, 65, 997

${ }^{16}$ Kanno, O.; Kawamoto, I. Tetrahedron 2000, 56, 5639

${ }^{17}$ Swift G.; Swern D. J. Org. Chem., 1967, 32, 511.

${ }^{18}$ Yamaguchi, M.; Hirao, I. Tetrahedron Lett. 1983, 24, 391-394.

${ }^{19}$ Obtained by reduction of enantiopure methyl (S)-3-amino-3-phenylpropionate ( $\geq 99 \%$ ee; assayed by HPLC using $10 \%$ isopropanol-hexane at $1 \mathrm{~mL} / \mathrm{min}$ on OD-H CHIRALCEL column)
} 
(m, 1H); ${ }^{13} \mathrm{C}$ NMR (75 MHz, CDCl3): 162.9, 142.5, 129.0, 127.8, 126.7, 59.5, 58.4, 56.1, 40.3, 35.5; IR (KBr, cm $\left.{ }^{-1}\right)$ 2940, 2854, 1616, 700; MS: HR-FAB calculated for $\mathrm{C}_{12} \mathrm{H}_{16} \mathrm{~N}_{2} \mathrm{OS}\left(\mathrm{M}^{+}\right)$ $\mathrm{m} / \mathrm{z}$ : 243.1143, Found: $243.1134 ; \mathbf{m p} 124-125^{\circ} \mathrm{C} ;[\alpha]_{\mathbf{D}}=-92.0^{\circ}(c 1.00, \mathrm{MeOH})$.

\section{(S)-Homotetramisole (S-HTM, 15)}

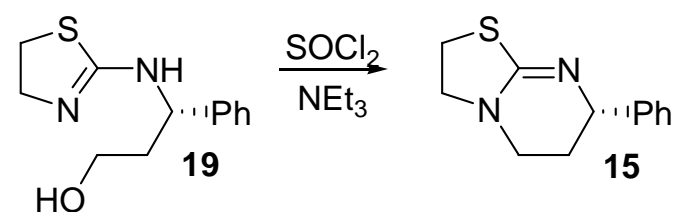

Thionyl chloride $(0.11 \mathrm{~mL}, 1.5 \mathrm{mmol})$ was added into a solution of $0.168 \mathrm{~g}(0.71 \mathrm{mmol})$ of 19 in anhydrous $\mathrm{CHCl}_{3}(2 \mathrm{~mL})$. The reaction mixture was refluxed for $1.5 \mathrm{~h}$ and then quenched by adding a few drops of $\mathrm{MeOH}$. After evaporation of the solvent, $10 \% \mathrm{NaOH}(10 \mathrm{~mL})$ was added to the residue, followed by extraction of $\mathrm{CH}_{2} \mathrm{Cl}_{2}(20 \mathrm{~mL}, 3$ times $)$. The combined organic extract was washed with brine, dried over $\mathrm{Na}_{2} \mathrm{SO}_{4}$ and rotary evaporated. Flash chromatography $(20 \% i-$ $\mathrm{PrOH}, 5 \% \mathrm{NEt}_{3} /$ hexanes) afforded $0.114 \mathrm{~g}$ of $\mathbf{1 5}$ as a yellow solid (74\% yield).

${ }^{1} \mathbf{H}$ NMR $\left(300 \mathrm{MHz}, \mathrm{CDCl}_{3}\right): \delta$ 7.35-7.18 (m, 5H), $4.56\left(\mathrm{dd}, J_{1}=4.4 \mathrm{~Hz}, J 2=7.9 \mathrm{~Hz}, 1 \mathrm{H}\right), 3.64-$ $3.50(\mathrm{~m}, 2 \mathrm{H}), 3.35-3.27(\mathrm{~m}, 1 \mathrm{H}), 3.20-3.11(\mathrm{~m}, 3 \mathrm{H}), 2.20-2.11(\mathrm{~m}, 1 \mathrm{H}), 1.89-1.77(\mathrm{~m}, 1 \mathrm{H})$;

${ }^{13}$ CNMR (75 MHz, $\left.\mathrm{CDCl}_{3}\right): \delta 161.0,145.2,128.5,126.8,126.7,58.3,54.0,43.5,29.3,26.6$; IR $\left(\mathrm{KBr}, \mathrm{cm}^{-1}\right)$ 2925, 2852, 1616, 1287, 701; MS: HR-FAB calculated for $\mathrm{C}_{12} \mathrm{H}_{14} \mathrm{~N}_{2} \mathrm{~S}\left(\mathrm{M}^{+}\right) \mathrm{m} / \mathrm{z}$ : 225.1038, Found: 225.1034 ; $\mathbf{m p} 106-107^{\circ} \mathrm{C} ;[\alpha]_{\mathbf{D}}=-132.5^{\circ}(c 0.83, \mathrm{MeOH})$.

\section{Preparation of $(S)$-HBTM}

(S)-3-(2-Benzothiazolylamino)-3-phenylpropanol (21)

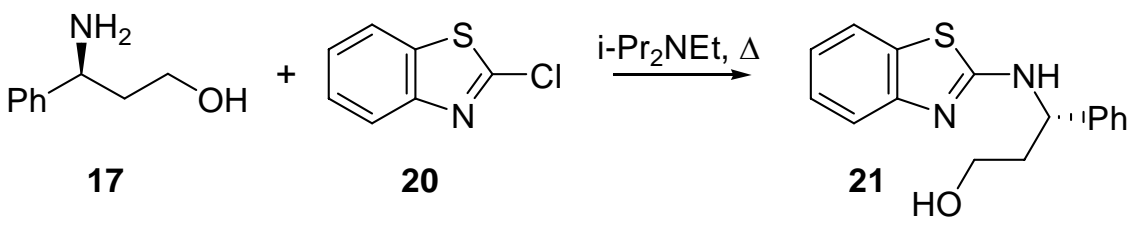

A 25-mL pressure tube charged with (S)-3-amino-3-phenylpropanol $\mathbf{1 7}^{6}(0.307 \mathrm{~g}, 2.0 \mathrm{mmol})$, 2chlorobenzothiazole $20(0.378 \mathrm{~g}, 2.2 \mathrm{mmol}), i$ - $\operatorname{Pr}_{2} \mathrm{NEt}(0.70 \mathrm{~mL}, 4.0 \mathrm{mmol})$ and a stir bar was flushed with nitrogen several times, stoppered and heated at $135 \pm 5{ }^{\circ} \mathrm{C}$ overnight. After cooling the tube to $30-40{ }^{\circ} \mathrm{C}$, the viscous reaction mixture was treated with $2 \mathrm{~mL}$ of $\mathrm{CH}_{2} \mathrm{Cl}_{2}$ and left at room temperature to dissolve. The diluted reaction mixture was applied directly to a chromatographic column and eluted with $8 \%$ isopropanol and $2.5 \% \mathrm{NEt}_{3}$ in hexane to afford $0.466 \mathrm{~g}$ of 21 as a white solid (77\% yield).

${ }^{1} \mathbf{H}$ NMR $\left(300 \mathrm{MHz}, \mathrm{CDCl}_{3}\right): \delta 7.53(\mathrm{~d}, J=8.1 \mathrm{~Hz}, 2 \mathrm{H}), 7.43-7.27(\mathrm{~m}, 6 \mathrm{H}), 7.08(\mathrm{t}, J=8.1 \mathrm{~Hz}$, $1 \mathrm{H}), 5.87$ (br, $1 \mathrm{H}), 5.23-5.17(\mathrm{~m}, 1 \mathrm{H}), 4.32$ (br, $1 \mathrm{H}), 3.80-3.78(\mathrm{~m}, 2 \mathrm{H}), 2.28-2.17(\mathrm{~m}, 1 \mathrm{H}), 2.07-$ $1.97(\mathrm{~m}, 1 \mathrm{H}) ;{ }^{13} \mathrm{C}$ NMR $\left(75 \mathrm{MHz}, \mathrm{CDCl}_{3}\right): \delta 167.6,151.7,141.6,130.5,129.2,128.1,126.9$, 126.2, 122.1, 121.0, 119.1, 58.9, 57.6, 39.8; IR (KBr, cm-1) 3220, 2923, 1598, 1550, 1448; MS: 
HR-FAB calculated for $\mathrm{C}_{16} \mathrm{H}_{16} \mathrm{~N}_{2} \mathrm{OS}\left(\mathrm{M}^{+}\right) \mathrm{m} / \mathrm{z}$ : 291.1143, Found: 291.1149 ; mp 166-167 ${ }^{\circ} \mathrm{C}$; $[\alpha]_{\mathbf{D}}=+64.5 .0^{\circ}(c 0.69, \mathrm{MeOH})$.

\section{(S)-Homobenzotetramisole (S-HBTM, 16)}

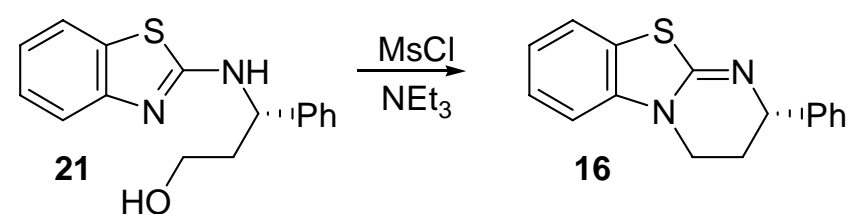

A solution of $0.446 \mathrm{~g}(1.57 \mathrm{mmol})$ of 21 in anhydrous $\mathrm{CH}_{2} \mathrm{Cl}_{2}(16 \mathrm{~mL})$ was cooled to $0{ }^{\circ} \mathrm{C}$ under $\mathrm{N}_{2}$ atmosphere and treated with $\mathrm{NEt}_{3}(0.66 \mathrm{~mL}, 4.7 \mathrm{mmol})$ followed by $\mathrm{MsCl}(0.24 \mathrm{~mL}, 3.1$ $\mathrm{mmol})$. The mixture was stirred at $0{ }^{\circ} \mathrm{C}$ for $1 \mathrm{~h}$ and then warmed up to room temperature. Methanol $(0.2 \mathrm{~mL})$ was then added to quench the excess $\mathrm{MsCl}_{\mathrm{NEt}} \mathrm{NE}_{3}(1.5 \mathrm{~mL})$ was added and the mixture was refluxed overnight. The cooled mixture was washed with a small amount of water, dried over $\mathrm{Na}_{2} \mathrm{SO}_{4}$ and rotary evaporated. The crude product was purified by chromatography ( $4 \% \mathrm{i}-\mathrm{PrOH}, 1 \% \mathrm{NEt}_{3} /$ hexanes) to give $0.36 \mathrm{~g}$ of $\mathbf{1 6}$ as a white solid ( $86 \%$ yield).

${ }^{1}$ H NMR $\left(300 \mathrm{MHz}, \mathrm{CDCl}_{3}\right): \delta 7.35-7.17(\mathrm{~m}, 5 \mathrm{H}), 7.01(\mathrm{t}, J=7.6 \mathrm{~Hz}, 1 \mathrm{H}), 6.74(\mathrm{~d}, J=7.6 \mathrm{~Hz}$, $1 \mathrm{H}), 4.72\left(\mathrm{dd}, J_{1}=4.0 \mathrm{~Hz}, J_{2}=12.0 \mathrm{~Hz}, 1 \mathrm{H}\right), 3.86-3.78(\mathrm{~m}, 1 \mathrm{H}), 3.72-3.64(\mathrm{~m}, 1 \mathrm{H}), 2.35-2.26$ $(\mathrm{m}, 1 \mathrm{H}), 2.04-1.92(\mathrm{~m}, 1 \mathrm{H}) ;{ }^{13} \mathbf{C}$ NMR $\left(75 \mathrm{MHz}, \mathrm{CDCl}_{3}\right): \delta 158.3,144.4,140.9,128.7,127.1$, 126.8, 126.1, 122.8, 122.1, 122.0, 107.6, 58.6, 40.7, 28.1; IR (KBr, cm $\left.{ }^{-1}\right) 1618,1579,1478$; MS: HR-FAB calculated for $\mathrm{C}_{16} \mathrm{H}_{14} \mathrm{~N}_{2} \mathrm{~S}(\mathrm{M}+) \mathrm{m} / \mathrm{z}$ : 273.1038, Found: 273.1047 ; mp $140-141{ }^{\circ} \mathrm{C}$; $[\alpha]_{\mathbf{D}}$ $=+82.4^{\circ}(c 1.00, \mathrm{MeOH})$.

\section{Preparation of new substrates.}

Note: The following procedures have been performed only once and have not been optimized.

trans-2-(2-Thienyl)-cyclohexanol (26)

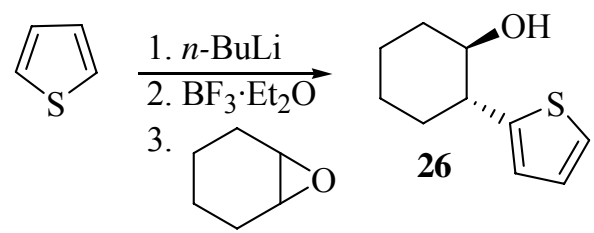

$n$ Butyllithium $(2.0 \mathrm{M}, 2.0 \mathrm{~mL}$ ) was added dropwise to a solution of thiophene (258 $\mathrm{mg}, 3.1$ $\mathrm{mmol})$ in THF $(15 \mathrm{~mL})$ at -78 under nitrogen atmosphere. Boron trifluoride etherate $(0.5 \mathrm{~mL})$ was added after $30 \mathrm{~min}$. After the reaction mixture was stirred for another $15 \mathrm{~min}$, cyclohexene oxide $(0.36 \mathrm{~mL}, 3.7 \mathrm{mmol})$ was added slowly. The reaction was quenched after $2.5 \mathrm{~h}$ with $10 \mathrm{~mL}$ of saturated aqueous $\mathrm{NH}_{4} \mathrm{Cl}$ solution. The mixture was extracted with $\mathrm{Et}_{2} \mathrm{O}$, the extract was dried over $\mathrm{MgSO}_{4}$ and rotary evaporated. The crude product was purified by chromatography $(20 \%$ $\mathrm{Et}_{2} \mathrm{O} /$ hexanes) to give $0.218 \mathrm{~g}$ of $\mathbf{2 6}$ as an oil (39\% yield).

${ }^{1} \mathbf{H}$ NMR $\left(300 \mathrm{MHz}, \mathrm{CDCl}_{3}\right): \delta 7.20(\mathrm{~d}, J=3.6 \mathrm{~Hz}, 1 \mathrm{H}), 6.97\left(\mathrm{dd}, J_{1}=3.6 \mathrm{~Hz}, J_{2}=3.3 \mathrm{~Hz}, 1 \mathrm{H}\right)$, $6.92\left(\mathrm{dd}, J_{1}=3.3 \mathrm{~Hz}, J_{2}=9.9 \mathrm{~Hz}, 1 \mathrm{H}\right), 3.49\left(\mathrm{dt}, J_{1}=4.4 \mathrm{~Hz}, J 2=9.9 \mathrm{~Hz}, 1 \mathrm{H}\right) 2.75\left(\mathrm{ddd}, J_{1}=4.4\right.$ $\left.\mathrm{Hz}, J 2=9.9 \mathrm{~Hz}, J_{3}=12.4 \mathrm{~Hz}, 1 \mathrm{H}\right), 2.14-1.99(\mathrm{~m}, 2 \mathrm{H}), 1.87-1.73(\mathrm{~m}, 3 \mathrm{H}), 1.61-1.26(\mathrm{~m}, 4 \mathrm{H}) ;{ }^{13} \mathrm{C}$ 
NMR (75 MHz, $\left.\mathrm{CDCl}_{3}\right): \delta 147.3,127.0,124.6,123.9,75.6,48.5,34.4,34.3,26.2,25.0 ; \mathbf{I R}$ $\left(\mathrm{KBr}, \mathrm{cm}^{-1}\right) 2928$ (br), 2854, 1447; MS: HR-ESI calculated for $\mathrm{C}_{10} \mathrm{H}_{14} \mathrm{OS}\left(\mathrm{M}+\mathrm{Na}^{+}\right) \mathrm{m} / \mathrm{z}$ : 205.0663, Found: 205.0668.

trans-2-(1H-indol-3-yl)-cyclohexanol (27)<smiles>O[C@H]1CCCC[C@@H]1c1c[nH]c2ccccc12</smiles>

$n$ Butyllithium $(2.5 \mathrm{M}, 0.8 \mathrm{~mL})$ was added dropwise to a solution of indole $(180 \mathrm{mg}, 1.5 \mathrm{mmol})$ in THF $(2.5 \mathrm{~mL})$ at $-78{ }^{\circ} \mathrm{C}$ under nitrogen atmosphere. Boron trifluoride etherate $(0.25 \mathrm{~mL})$ was added after $30 \mathrm{~min}$. After the reaction mixture was stired for another $15 \mathrm{~min}$, cyclohexene oxide $(0.19 \mathrm{~mL}, 0.97 \mathrm{mmol})$ was added slowly. The reaction was quenched after $2.5 \mathrm{~h}$ with $10 \mathrm{~mL}$ of saturated $\mathrm{NH}_{4} \mathrm{Cl}$ solution. The separated aqueous phase was extracted with $\mathrm{Et}_{2} \mathrm{O}$, dried over $\mathrm{MgSO}_{4}$ and rotary evaporated. The crude product was purified by chromatography (20\% EtOAc/hexanes) to give $0.114 \mathrm{~g}$ of 27 as a white solid (34\% yield).

${ }^{1} \mathbf{H}$ NMR $\left(300 \mathrm{MHz}, \mathrm{CDCl}_{3}\right): \delta 8.15(\mathrm{br}, 1 \mathrm{H}), 7.73(\mathrm{~d}, J=8.0 \mathrm{~Hz}, 1 \mathrm{H}), 7.36(\mathrm{~d}, J=8.2 \mathrm{~Hz}, 1 \mathrm{H})$, $7.20(\mathrm{t}, J=7.1 \mathrm{~Hz}, 1 \mathrm{H}), 7.12(\mathrm{t}, J=7.1 \mathrm{~Hz}, 1 \mathrm{H}), 7.07(\mathrm{~d}, J=2.5 \mathrm{~Hz}, 1 \mathrm{H}), 3.78\left(\mathrm{dt}, J_{1}=4.4 \mathrm{~Hz}\right.$, $\left.J_{2}=10.2 \mathrm{~Hz}, 1 \mathrm{H}\right), 2.76\left(\mathrm{ddd}, J_{1}=4.4 \mathrm{~Hz}, J_{2}=9.9 \mathrm{~Hz}, J_{3}=12.4 \mathrm{~Hz}, 1 \mathrm{H}\right), 2.19-1.64(\mathrm{~m}, 7 \mathrm{H}), 1.51-$ 1.35 (m, 2H); ${ }^{13} \mathbf{C}$ NMR (75 MHz, $\left.\mathrm{CDCl}_{3}\right): \delta 136.8,127.3,122.4,121.6,119.8,119.6,117.8$, 111.5, 75.0,44.7, 34.5, 32.9, 26.5, 25.3; IR (KBr, cm $\left.{ }^{-1}\right) 2928$ (br), 3410, 3299, 2928, 2855, 1456; MS: HR-ESI calculated for $\mathrm{C}_{14} \mathrm{H}_{17} \mathrm{NO}\left(\mathrm{M}+\mathrm{Na}^{+}\right) \mathrm{m} / \mathrm{z}$ : 238.1208, Found: 238.1204. Mp 154-156 ${ }^{\circ} \mathrm{C}$. 


\section{Characterization data and HPLC properties of cycloalkanol substrates and their acylated derivatives.}

Note: The absolute configurations of cycloalkanols shown below correspond to the predominant enantiomers obtained by KR using the (S)-enantiomer of catalyst $\mathbf{1 6}$.

\begin{tabular}{|c|c|}
\hline 24 & $\begin{array}{l}\text { HPLC (CHIRALCEL OD-H, } 2.0 \% \text { IPA/hexane, } 1.0 \mathrm{~mL} / \mathrm{min}) \text { : (S)- } \\
\text { enantiomer: } 11.2 \mathrm{~min} \text {; (R)-enantiomer: } 12.7 \mathrm{~min} . \\
\text { The absolute configuration of unreacted }(1 \mathrm{~S}, 2 R)-(+)-24 \text { was assigned by } \\
\text { comparison of the sign of optical rotation with literature data }{ }^{20} .\end{array}$ \\
\hline Propion & $\begin{array}{l}{ }^{1} \mathbf{H} \text { NMR }\left(300 \mathrm{MHz}, \mathrm{CDCl}_{3}\right): \delta 7.28-7.14(\mathrm{~m}, 5 \mathrm{H}), 4.97\left(\mathrm{dt}, J_{1}=4.4 \mathrm{~Hz},\right. \\
\left.J_{2}=10.7 \mathrm{~Hz}, 1 \mathrm{H}\right), 2.65\left(\mathrm{dt}, J_{1}=4.4 \mathrm{~Hz}, J_{2}=11.5 \mathrm{~Hz}, 1 \mathrm{H}\right), 2.12-1.75(\mathrm{~m}, 6 \mathrm{H}), \\
1.67-1.31(\mathrm{~m}, 4 \mathrm{H}), 0.83(\mathrm{t}, J=7.5 \mathrm{~Hz}, 3 \mathrm{H}) ;{ }^{13} \mathrm{C} \mathbf{N M R}\left(75 \mathrm{MHz}, \mathrm{CDCl}_{3}\right): \\
\delta 173.9,143.3,128.4,127.7,126.5,75.9,50.1,34.0,32.6,27.9,26.0,25.0, \\
9.2 ; \mathbf{I R}\left(\mathrm{KBr}, \mathrm{cm}^{-1}\right) 2936,1732,1189 ; \mathbf{M S}: \text { HR-ESI calculated for } \\
\mathrm{C}_{15} \mathrm{H}_{20} \mathrm{NO}_{2}\left(\mathrm{M}+\mathrm{H}^{+}\right) \mathrm{m} / \mathrm{z}: 233.1542 \text {, Found: } 233.1543 \\
\text { HPLC analysis was performed on the corresponding alcohol } 24 \text { obtained by } \\
\text { base hydrolysis. }\end{array}$ \\
\hline & $\begin{array}{l}\text { HPLC (CHIRALCEL OD-H, } 1.5 \% \mathrm{IPA} / \text { hexane, } 0.8 \mathrm{~mL} / \mathrm{min}) \text { : (S)- } \\
\text { enantiomer: } 16.0 \mathrm{~min} \text {; (R)-enantiomer: } 17.9 \mathrm{~min} \text {. } \\
\text { The absolute configuration of unreacted }(1 \mathrm{~S}, 2 \mathrm{~S})-(+)-26 \text { was analyzed by the } \\
\text { Mosher method }^{21} \text {. }\end{array}$ \\
\hline & $\begin{array}{l}{ }^{1} \mathrm{H} \text { NMR }\left(300 \mathrm{MHz}, \mathrm{CDCl}_{3}\right): \delta 7.12\left(\mathrm{ddd}, J_{1}=1.1 \mathrm{~Hz}, J_{2}=3.3 \mathrm{~Hz}, J_{3}=4.7\right. \\
\mathrm{Hz}, 1 \mathrm{H}), 6.90\left(\mathrm{ddd}, J_{1}=1.1 \mathrm{~Hz}, J_{2}=3.3 \mathrm{~Hz}, J_{3}=4.7 \mathrm{~Hz}, 1 \mathrm{H}\right), 6.82\left(\mathrm{ddd}, J_{1}=\right. \\
\left.1.1 \mathrm{~Hz}, J_{2}=3.3 \mathrm{~Hz}, J_{3}=4.7 \mathrm{~Hz}, 1 \mathrm{H}\right), 4.80\left(\mathrm{dt}, J_{1}=4.4 \mathrm{~Hz}, J_{2}=10.2 \mathrm{~Hz}, 1 \mathrm{H}\right), \\
2.95\left(\mathrm{dt}, J_{1}=4.4 \mathrm{~Hz}, J_{2}=13.2 \mathrm{~Hz}, 1 \mathrm{H}\right), 2.22-2.09(\mathrm{~m}, 4 \mathrm{H}), 1.85-1.31(\mathrm{~m}, 6 \mathrm{H}) \text {, } \\
0.98(\mathrm{t}, J=7.6 \mathrm{~Hz}, 3 \mathrm{H}) ;{ }^{13} \mathrm{C} \mathrm{NMR}\left(75 \mathrm{MHz}, \mathrm{CDCl}_{3}\right): \delta 173.8,146.7,126.5, \\
123.8,123.2,76.7,44.5,34.2,32.3,28.0,25.8,24.6,9.2 ; \mathbf{I R}\left(\mathrm{KBr}, \mathrm{cm}^{-1}\right) \\
\text { 2936, 2858, 1733, } 1185 ; \mathbf{M S}: \mathrm{HR}-\mathrm{ESI} \text { calculated for } \mathrm{C}_{13} \mathrm{H}_{15} \mathrm{NO}_{3}\left(\mathrm{M}+\mathrm{Na}^{+}\right) \\
\text {m/z: } 261.0925 \text {, Found: } 261.0922 ; \\
\text { HPLC analysis was performed on the corresponding alcohol } 26 \text { obtained by } \\
\text { base hydrolysis. }\end{array}$ \\
\hline 27 & $\begin{array}{l}\text { HPLC (CHIRALCEL OD-H, } 10 \% \mathrm{IPA} / \text { hexane, } 1.0 \mathrm{~mL} / \mathrm{min}) \text { : (S)- } \\
\text { enantiomer: } 20.5 \mathrm{~min} \text {; (R)-enantiomer: } 25.1 \mathrm{~min} \text {. } \\
\text { The absolute configuration of unreacted }(1 \mathrm{~S}, 2 R)-(+)-27 \text { was analyzed by the } \\
\text { Mosher method }^{7} \text {. }\end{array}$ \\
\hline
\end{tabular}

\footnotetext{
${ }^{20}$ Brown, H. C.; Prasad J. V. N. V.; Gupta, A. K.; Bakshi R. K. J. Org. Chem. 1987, 52, 310.

${ }^{21}$ Ohtani I.; Kusumi T.; Kashman Y.; Kakisawa H. J. Am. Chem. Soc. 1991, 113, 4092.
} 


\begin{tabular}{|c|c|}
\hline Propionate of 27 & 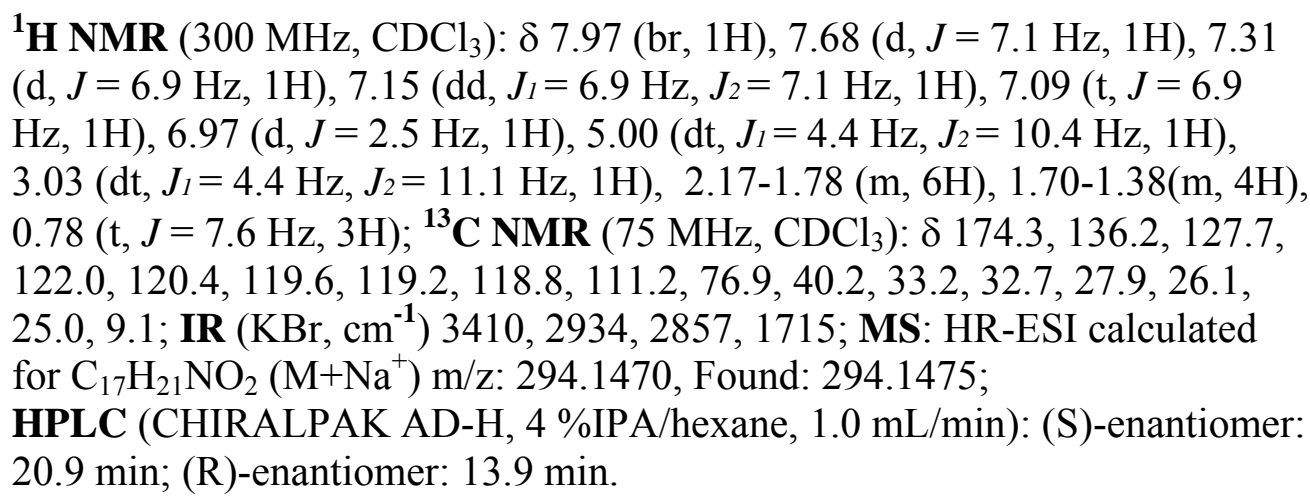 \\
\hline 28 & $\begin{array}{l}\text { HPLC (CHIRALCEL OD-H, } 1.5 \% \mathrm{IPA} / \text { hexane, } 1.0 \mathrm{~mL} / \mathrm{min}) \text { : }(\mathrm{S})- \\
\text { enantiomer: } 19.5 \mathrm{~min} \text {; }(\mathrm{R}) \text {-enantiomer: } 22.7 \mathrm{~min} \text {. } \\
\text { The absolute configuration of unreacted }(1 S, 2 R)-(+)-28 \text { was assigned by } \\
\text { comparison of the sign of optical rotation with literature data. }\end{array}$ \\
\hline Propionate of $\mathbf{2 8}$ & $\begin{array}{l}{ }^{1} \mathbf{H} \text { NMR }\left(300 \mathrm{MHz}, \mathrm{CDCl}_{3}\right): \delta 7.31-7.17(\mathrm{~m}, 5 \mathrm{H}), 5.12\left(\mathrm{dt}, J_{1}=5.2 \mathrm{~Hz},\right. \\
\left.J_{2}=6.6 \mathrm{~Hz}, 1 \mathrm{H}\right), 3.16\left(\mathrm{dt}, J_{1}=5.2 \mathrm{~Hz}, J_{2}=7.8 \mathrm{~Hz}, 1 \mathrm{H}\right), 2.31-2.13(\mathrm{~m}, 4 \mathrm{H}), \\
1.89-1.65(\mathrm{~m}, 4 \mathrm{H}), 1.09(\mathrm{t}, J=7.6 \mathrm{~Hz}, 3 \mathrm{H}) ;{ }^{13} \mathbf{C ~ N M R}\left(75 \mathrm{MHz}, \mathrm{CDCl}_{3}\right) \text { : } \\
\delta 174.4,143.0,128.6,127.4,126.5,81.9,51.2,32.2,32.1,28.0,23.1,9.3 ; \mathbf{I R} \\
\left(\mathrm{KBr}, \mathrm{cm}^{-1}\right) 2963,1734,1189 ; \mathbf{M S}: \mathrm{HR}-\mathrm{ESI} \text { calculated for } \mathrm{C}_{14} \mathrm{H}_{18} \mathrm{O}_{2}\left(\mathrm{M}^{-} \mathrm{H}^{+}\right) \\
\text {m/z: } 219.1385, \text { Found: } 219.1383 ; \\
\text { HPLC analysis was performed on the corresponding alcohol } 28 \text { obtained by } \\
\text { base hydrolysis. }\end{array}$ \\
\hline 29 & $\begin{array}{l}\text { HPLC (CHIRALCEL OD-H, } 1.5 \% \text { IPA/hexane, } 1.0 \mathrm{~mL} / \mathrm{min}):(\mathrm{S})- \\
\text { enantiomer: } 9.5 \mathrm{~min} \text {; (R)-enantiomer: } 12.3 \mathrm{~min} \text {. } \\
\text { The absolute configuration of unreacted }(1 \mathrm{~S}, 2 \mathrm{~S})-(+)-29 \text { was assigned by } \\
\text { comparison of the sign of optical rotation with literature data }{ }^{23} \text {. }\end{array}$ \\
\hline Propionate of 29 & $\begin{array}{l}{ }^{1} \mathbf{H} \text { NMR }\left(300 \mathrm{MHz}, \mathrm{CDCl}_{3}\right): \delta 7.29-7.15(\mathrm{~m}, 5 \mathrm{H}), 5.16(\mathrm{~m}, 1 \mathrm{H}), 2.78\left(\mathrm{dt}, J_{1}\right. \\
\left.=3.0 \mathrm{~Hz}, J_{2}=12.6 \mathrm{~Hz}, 1 \mathrm{H}\right), 2.25-2.13(\mathrm{~m}, 2 \mathrm{H}), 2.10-2.01(\mathrm{~m}, 2 \mathrm{H}), 1.93-1.89 \\
(\mathrm{~m}, 1 \mathrm{H}), 1.79-1.73(\mathrm{~m}, 1 \mathrm{H}), 1.69-1.41(\mathrm{~m}, 4 \mathrm{H}), 1.01(\mathrm{t}, J=7.6 \mathrm{~Hz}, 3 \mathrm{H}) ;{ }^{13} \mathrm{C} \\
\text { NMR }\left(75 \mathrm{MHz}, \mathrm{CDCl}_{3}\right): \delta 173.7,143.5,128.2,128.0,126.5,73.1,46.7 \text {, } \\
30.9,28.1,26.1,20.4,9.3 ; \mathbf{I R}\left(\mathrm{KBr}, \mathrm{cm}^{-1}\right) 2936,1732,1194,1177 ; \mathbf{M S}: \mathrm{HR}- \\
\text { ESI calculated for } \mathrm{C}_{15} \mathrm{H}_{20} \mathrm{O}_{2}\left(\mathrm{M}+\mathrm{H}^{+}\right) \mathrm{m} / \mathrm{z}: 233.1542 \text {, Found: } 233.1543 ; \\
\text { HPLC analysis was performed on the corresponding alcohol } 29 \text { obtained by } \\
\text { base hydrolysis. }\end{array}$ \\
\hline
\end{tabular}

\footnotetext{
22 Mandal, A. K.; Jadhav, P. K.; Brown, H. C. J. Org. Chem. 1980, 45, 3543.

${ }^{23}$ Peach, P.; Cross, D. J.; Kenny, J. A.; Mann, I.; Houson, I.; Campbell, L.; Walsgrove, T.; Wills, M. Tetrahedron 2006, 62, 1864.
} 


\begin{tabular}{|c|c|}
\hline $\mathrm{OH}$ & $\begin{array}{l}\text { HPLC (CHIRALCEL AS-H, } 10 \% \mathrm{IPA} / \text { hexane, } 1.0 \mathrm{~mL} / \mathrm{min}) \text { : }(\mathrm{S})- \\
\text { enantiomer: } 8.2 \mathrm{~min} \text {; (R)-enantiomer: } 15.8 \mathrm{~min} \text {. } \\
\text { The absolute configuration of unreacted }(1 \mathrm{~S}, 2 \mathrm{~S})-(+)-30 \text { was assigned by } \\
\text { comparison of the sign of optical rotation with literature data }{ }^{24} \text {. }\end{array}$ \\
\hline $\begin{array}{l}\text { 'OBz } \\
\text { Propionate of } \mathbf{3 0}\end{array}$ & $\begin{array}{l}{ }^{1} \mathbf{H} \text { NMR }\left(300 \mathrm{MHz}, \mathrm{CDCl}_{3}\right): \delta 8.01(\mathrm{t}, J=8.2 \mathrm{~Hz}, 2 \mathrm{H}), 7.54(\mathrm{t}, J=8.8 \mathrm{~Hz}, \\
1 \mathrm{H}), 7.43\left(\mathrm{dd}, \mathrm{J}_{1}=8.2 \mathrm{~Hz}, J_{2}=8.8 \mathrm{~Hz}, 2 \mathrm{H}\right), 5.10-4.98(\mathrm{~m}, 2 \mathrm{H}), 2.29-2.15(\mathrm{~m}, \\
4 \mathrm{H}), 1.79-1.72(\mathrm{~m}, 2 \mathrm{H}), 1.58-1.38(\mathrm{~m}, 4 \mathrm{H}), 1.00(\mathrm{t}, J=7.6 \mathrm{~Hz}, 3 \mathrm{H}) ;{ }^{13} \mathrm{C} \\
\text { NMR }\left(75 \mathrm{MHz}, \mathrm{CDCl}_{3}\right): \delta 174.1,166.1,133.1,130.4,129.8,128.5,74.8, \\
\left.73.4,30.2,30.4,28.0,23.71,23.68,9.3 ; \mathrm{IR}_{\left(\mathrm{KBr}, \mathrm{cm}^{-1}\right)}\right) 2942,2866,1736, \\
\text { 1720, 1274; MS: HR-ESI calculated for } \mathrm{C}_{16} \mathrm{H}_{20} \mathrm{O}_{4}\left(\mathrm{M}+\mathrm{Na}^{+}\right) \mathrm{m} / \mathrm{z}: 299.1259 \text {, } \\
\text { Found: } 299.1268 ; \\
\text { HPLC (CHIRALPAK AD-H, } 1 \% \mathrm{IPA} / \text { hexane, } 1.0 \mathrm{~mL} / \mathrm{min}):(\mathrm{S}) \text {-enantiomer: } \\
\text { 9.8 min; (R)-enantiomer: } 11.2 \mathrm{~min} \text {. }\end{array}$ \\
\hline & $\begin{array}{l}\text { HPLC (CHIRALCEL OD-H, } 2 \% \mathrm{IPA} / \text { hexane, } 1.0 \mathrm{~mL} / \mathrm{min}) \text { : (S)-enantiomer: } \\
13.7 \mathrm{~min} \text {; (R)-enantiomer: } 11.4 \mathrm{~min} \text {. } \\
\text { The absolute configuration of unreacted }(1 S, 2 S)-(+)-31 \text { was assigned by } \\
\text { comparison of the sign of optical rotation with literature data }{ }^{25} \text {. }\end{array}$ \\
\hline $\begin{array}{l}{ }^{\prime} \mathrm{CO}_{2} \mathrm{Et} \\
\text { Propionate of } 31\end{array}$ & 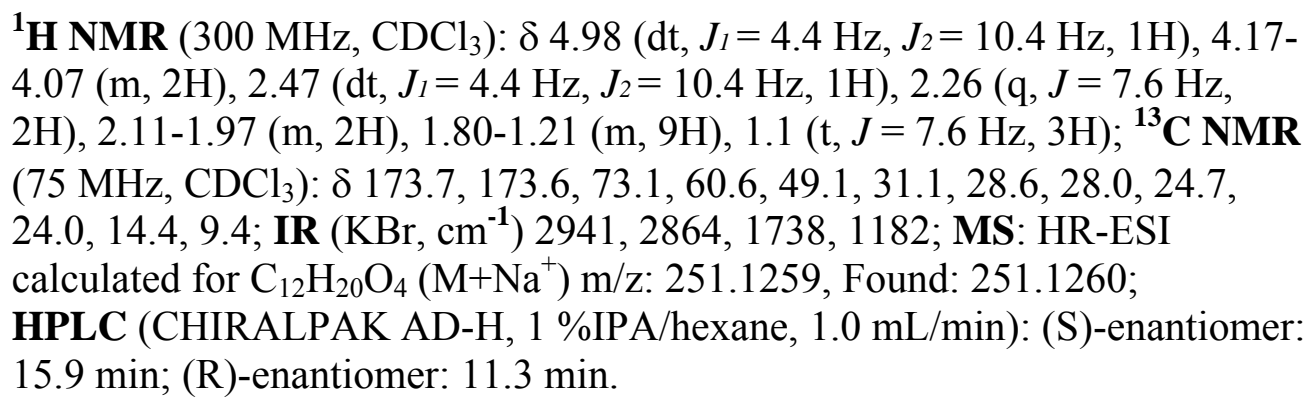 \\
\hline $\mathrm{N}_{3}$ & $\begin{array}{l}\text { HPLC (CHIRALCEL OD-H, } 0.5 \% \text { IPA/hexane, } 0.8 \mathrm{~mL} / \mathrm{min}) \text { : }(\mathrm{S})- \\
\text { enantiomer: } 30.2 \mathrm{~min} \text {; (R)-enantiomer: } 31.9 \mathrm{~min} \text {. } \\
\text { The absolute configuration of unreacted }(1 \mathrm{~S}, 2 \mathrm{~S})-(+)-32 \text { was assigned by } \\
\text { comparison of the sign of optical rotation with literature data }{ }^{26} \text {. }\end{array}$ \\
\hline Propionate of 32 & $\begin{array}{l}{ }^{1} \mathbf{H} \text { NMR }\left(300 \mathrm{MHz}, \mathrm{CDCl}_{3}\right): \delta 4.74-4.66(\mathrm{~m}, 1 \mathrm{H}), 3.43-3.46(\mathrm{~m}, 1 \mathrm{H}), \\
2.37(\mathrm{q}, J=7.6 \mathrm{~Hz}, 2 \mathrm{H}), 2.10-2.02(\mathrm{~m}, 2 \mathrm{H}), 1.76-1.72(\mathrm{~m}, 2 \mathrm{H}), 1.42-1.24(\mathrm{~m}, \\
4 \mathrm{H}), 1.17(\mathrm{t}, J=7.6 \mathrm{~Hz}, 3 \mathrm{H}) ;{ }^{13} \mathrm{C} \mathrm{NMR}\left(75 \mathrm{MHz}, \mathrm{CDCl}_{3}\right): \delta 173.9,75.5, \\
63.5,30.8,28.0,24.0,23.7,9.2 ; \mathbf{I R}\left(\mathrm{KBr}, \mathrm{cm}^{-1}\right) 2942,2096,1738 ; \mathrm{MS}: \mathrm{HR}- \\
\text { ESI calculated for } \mathrm{C}_{13} \mathrm{H}_{15} \mathrm{NO}_{3}\left(\mathrm{M}+\mathrm{Na}^{+}\right) \mathrm{m} / \mathrm{z}: 220.1062 \text {, Found: } 220.1055 ; \\
\text { HPLC analysis was performed on the corresponding alcohol } 32 \text { obtained by } \\
\text { base hydrolysis. }\end{array}$ \\
\hline
\end{tabular}

\footnotetext{
${ }^{24}$ Hu, G.; Vasella, A. Helv. Chim. Acta 2002, 85, 4369.

${ }^{25}$ Levy, L. M.; Dehli, J. R.; Gotor, V. Tetrahedron: Asymmetry, 2003, 14, 2053.

${ }^{26}$ Arndt, H.-D.; Ziemer, B.; Koert, U. Org. Lett. 2004, 6, 3269.
} 


\begin{tabular}{|c|c|}
\hline 33 & $\begin{array}{l}\text { HPLC analysis was performed on the benzoate ester: (CHIRALPAK AD-H, } \\
\text { pure hexane, } 1.0 \mathrm{~mL} / \mathrm{min}) \text { : (S)-enantiomer: } 9.8 \mathrm{~min} \text {; (R)-enantiomer: } 13.8 \\
\text { min. } \\
\text { The absolute configuration of unreacted }(1 \mathrm{~S}, 2 \mathrm{R}, 5 \mathrm{~S})-(+)-33 \text { was assigned by } \\
\text { HPLC comparison with the benzoate ester of commercial }(1 R, 2 S, 5 R)-(-)- \\
\text { menthol. }\end{array}$ \\
\hline $\mathrm{Ph}_{\mathrm{OH}}^{\mathrm{Me}} 34$ & $\begin{array}{l}\text { HPLC (CHIRALCEL OD-H, } 1.5 \% \text { IPA/hexane, } 1.0 \mathrm{~mL} / \mathrm{min}):(\mathrm{S})- \\
\text { enantiomer: } 14.1 \mathrm{~min} \text {; (R)-enantiomer: } 16.2 \mathrm{~min} . \\
\text { The absolute configuration of unreacted }(S)-(+)-35 \text { was assigned by } \\
\text { comparison of the sign of optical rotation with literature data }{ }^{27} \text {. }\end{array}$ \\
\hline
\end{tabular}

\section{KINETIC RESOLUTION EXPERIMENTS.}

Procedure A: Enantioselectivity test with ( \pm )-1-phenylpropanol.

The stock solutions of catalysts containing $0.050 \mathrm{mmol}$ of 3 , or $0.020 \mathrm{mmol}$ of 4 , or $0.005 \mathrm{mmol}$ of 15 or 16 and $65 \mu \mathrm{L}(0.375 \mathrm{mmol})$ of $i-\mathrm{Pr}_{2} \mathrm{NEt}$ in $2.00 \mathrm{~mL}$ of $\mathrm{CDCl}_{3}$ were prepared in the usual manner. A one-dram vial was charged with $( \pm)$-phenylpropanol $(34 \mathrm{mg}, 0.25 \mathrm{mmol})$ and $1.00 \mathrm{~mL}$ of a stock catalyst solution. Propionic anhydride $(24 \mu \mathrm{L}, 0.188 \mathrm{mmol})$ was added (at which point the timing was started), the content was mixed and transferred into a $5 \mathrm{~mm} \mathrm{NMR} \mathrm{tube.} \mathrm{The}$ reaction was monitored by ${ }^{1} \mathrm{H}$ NMR at room temperature by comparing integration values of the peaks at $\delta 4.5 \mathrm{ppm}$ and $\delta 5.6 \mathrm{ppm}$ and stopped by adding several drops of $\mathrm{MeOH}$ either after reaching $c a$. $50 \%$ conversion by NMR or when the reaction stopped $(<2 \%$ conversion increase in $0.5 \mathrm{~h})$ The workup and HPLC analysis followed the standard procedure. ${ }^{\text {a }}$

\section{Table 1S.}

\begin{tabular}{|c|c|c|c|c|c|c|c|c|c|}
\hline Entry & $\begin{array}{l}\text { catalyst } \\
(\mathrm{mol} \%)\end{array}$ & $\mathbf{t}(\mathbf{h})$ & \# & $\begin{array}{c}\text { ee }_{P R} \\
\%\end{array}$ & $\begin{array}{c}\mathbf{e e}_{\mathrm{SM}} \\
\%\end{array}$ & $\begin{array}{c}\mathrm{C}_{\text {HPLC }} \\
\%\end{array}$ & $\mathbf{S}$ & $\begin{array}{c}\mathrm{C}_{\mathrm{AVG}} \\
\% \\
\end{array}$ & $\mathbf{S}_{\mathrm{AVG}}$ \\
\hline \multirow{2}{*}{1} & \multirow{2}{*}{$3(10)$} & \multirow{2}{*}{3.0} & 1 & 88.3 & 50.1 & 36.2 & 26.5 & \multirow{2}{*}{31} & \multirow{2}{*}{28} \\
\hline & & & 2 & 90.8 & 32.6 & 26.4 & 28.6 & & \\
\hline \multirow{2}{*}{2} & \multirow{2}{*}{$4(4)$} & \multirow{2}{*}{2.2} & 1 & 92.8 & 86.3 & 48.2 & 75.3 & \multirow{2}{*}{49} & \multirow{2}{*}{72} \\
\hline & & & 2 & 91.7 & 88.7 & 49.2 & 68.5 & & \\
\hline \multirow{2}{*}{3} & \multirow{2}{*}{$15(1)$} & \multirow{2}{*}{1.7} & 1 & 86.3 & 76.8 & 47.1 & 31.5 & \multirow{2}{*}{47} & \multirow{2}{*}{30} \\
\hline & & & 2 & 84.6 & 77.1 & 47.7 & 27.8 & & \\
\hline \multirow{2}{*}{4} & \multirow{2}{*}{$16(1)$} & \multirow{2}{*}{1.5} & 1 & 83.6 & 77.1 & 48.0 & 25.9 & \multirow{2}{*}{48} & \multirow{2}{*}{26} \\
\hline & & & 2 & 83.6 & 77.0 & 48.0 & 25.9 & & \\
\hline
\end{tabular}

Procedure B: Enantioselectivity test with ( \pm )-trans-2-phenyl-cyclohexanol.

The stock solutions of catalysts containing $0.040 \mathrm{mmol}$ of 2 or $\mathbf{4}$, or $0.100 \mathrm{mmol}$ of 3, or 0.010 mmol of 15 or 16 and $65 \mu \mathrm{L}(0.375 \mathrm{mmol})$ of $i-\mathrm{Pr}_{2} \mathrm{NEt}$ in $2.00 \mathrm{~mL}$ of $\mathrm{CDCl}_{3}$ were prepared in

\footnotetext{
${ }^{27}$ Yadav, A. K.; Manju, M.; Chhinpa, P. R. Tetrahedron: Asymmetry 2003, 14, 1079.
} 
the usual manner. A one dram vial was charged with ( \pm )-trans-2-phenyl-cyclohexanol (44 mg, $0.25 \mathrm{mmol})$ and $1.00 \mathrm{~mL}$ of the stock solution of the catalyst. Propionic anhydride $(24 \mu \mathrm{L}, 0.188$ mmol) was added. The reaction was monitored by ${ }^{1} \mathrm{H}$ NMR at room temperature by comparing integration values of peaks at $\delta 3.6 \mathrm{ppm}$ and $\delta 4.9 \mathrm{ppm}$ and stopped by adding several drops of $\mathrm{MeOH}$ either after reaching $\mathrm{ca}$. $50 \%$ conversion by NMR or when the reaction stopped $(<2 \%$ conversion increase in $0.5 \mathrm{~h}$ ) The workup and HPLC analysis followed the standard procedure. ${ }^{\text {a }}$

Table 2S

\begin{tabular}{|c|c|c|c|c|c|c|c|c|c|}
\hline Entry & catalyst & anhydride & $\mathbf{t}(\mathbf{h})$ & $\begin{array}{c}\text { ee }_{P R} \\
\%\end{array}$ & $\begin{array}{c}\mathbf{e e}_{\mathrm{SM}} \\
\%\end{array}$ & $\begin{array}{c}\mathbf{C}_{\text {HPLC }} \\
\%\end{array}$ & $\mathbf{s}$ & $\begin{array}{c}\mathrm{C}_{\text {AVG }} \\
\%\end{array}$ & $\mathbf{S}_{\mathrm{AVG}}$ \\
\hline \multirow{3}{*}{1} & \multirow{3}{*}{$\begin{array}{c}\mathbf{4} \\
(8 \mathrm{~mol} \%)\end{array}$} & \multirow{3}{*}{$(\mathrm{EtCO})_{2} \mathrm{O}$} & \multirow{3}{*}{6} & 81.9 & 80.7 & 49.6 & 24.8 & \multirow{3}{*}{48} & \multirow{3}{*}{25} \\
\hline & & & & 84.6 & 71.2 & 45.7 & 25.4 & & \\
\hline & & & & 83.2 & 74.3 & 47.2 & 24.2 & & \\
\hline \multirow{2}{*}{2} & \multirow{2}{*}{$\begin{array}{c}3 \\
(20 \mathrm{~mol} \%)\end{array}$} & \multirow{2}{*}{$(\mathrm{EtCO})_{2} \mathrm{O}$} & \multirow{2}{*}{3} & 65.6 & 15.5 & 19.1 & 5.6 & \multirow{2}{*}{19} & \multirow{2}{*}{5.5} \\
\hline & & & & 65.0 & 15.1 & 18.8 & 5.5 & & \\
\hline \multirow{2}{*}{3} & \multirow{2}{*}{$\begin{array}{c}2 \\
(8 \mathrm{~mol} \%)\end{array}$} & \multirow{2}{*}{$(\mathrm{EtCO})_{2} \mathrm{O}$} & \multirow{2}{*}{12} & 74.7 & 57.9 & 43.7 & 12.3 & \multirow{2}{*}{45} & \multirow{2}{*}{11} \\
\hline & & & & 68.3 & 60.0 & 46.8 & 9.6 & & \\
\hline \multirow{2}{*}{4} & \multirow{2}{*}{$\begin{array}{c}15 \\
(2 \mathrm{~mol} \%)\end{array}$} & \multirow{2}{*}{$(\mathrm{EtCO}){ }_{2} \mathrm{O}$} & \multirow{2}{*}{1.8} & 76.0 & 60.3 & 44.2 & 13.5 & \multirow{2}{*}{43} & \multirow{2}{*}{14} \\
\hline & & & & 77.5 & 56.2 & 42.1 & 13.8 & & \\
\hline \multirow[t]{2}{*}{5} & \multirow{2}{*}{$\begin{array}{c}\mathbf{1 6} \\
(2 \mathrm{~mol} \%)\end{array}$} & \multirow{2}{*}{$(\mathrm{EtCO})_{2} \mathrm{O}$} & \multirow{2}{*}{1.4} & 84.0 & 82.2 & 49.5 & 29.2 & \multirow{2}{*}{47} & \multirow{2}{*}{29} \\
\hline & & & & 86.9 & 69.4 & 44.4 & 29.6 & & \\
\hline
\end{tabular}

Procedure C1: Variation of the type and amount of anhydride.

Four one-dram vial were charged with $( \pm$ )-trans-2-phenyl-cyclohexanol (44 mg, $0.25 \mathrm{mmol})$ and $1.00 \mathrm{~mL}$ of the stock solution of catalyst $16\left(0.005 \mathrm{M}\right.$ of 16 and $0.188 \mathrm{M}$ of $i-\operatorname{Pr}_{2} \mathrm{NEt}$ in $\left.\mathrm{CDCl}_{3}\right)$. Two more vials were prepared analogously, except that a reduced amount of $i-\operatorname{Pr}_{2} \mathrm{NEt}$ in the stock solution was used $(0.138 \mathrm{M})$. Aliquots of acetic anhydride $(17.7 \mu \mathrm{L}, 0.188 \mathrm{mmol})$ or isobutyric anhydride $(31 \mu \mathrm{L}, 0.188 \mathrm{mmol})$ were added to the four vials. Reduced aliquots of propionic anhydride $(17.6 \mu \mathrm{L}, 0.138 \mathrm{mmol})$ were added to the remaining two vials with reduced amounts of $i-\mathrm{Pr}_{2} \mathrm{NEt}$. The reactions were monitored by ${ }^{1} \mathrm{H} \mathrm{NMR}$ at room temperature and stopped by adding several drops of $\mathrm{MeOH}$ upon reaching $c a .50 \%$ conversion.

Table 3S

\begin{tabular}{|c|c|c|c|c|c|c|c|c|c|}
\hline Entry & anhydride & $\begin{array}{l}\text { Amount of } \\
\text { anhydride }\end{array}$ & $\mathbf{t}(\mathbf{h})$ & $\begin{array}{c}\mathbf{e e}_{\mathrm{PR}} \\
\%\end{array}$ & $\begin{array}{c}\mathbf{e e}_{\mathrm{SM}} \\
\%\end{array}$ & $\begin{array}{c}\mathrm{C}_{\text {HPLC }} \\
\%\end{array}$ & $\mathbf{S}$ & $\begin{array}{c}\mathrm{C}_{\mathrm{AVG}} \\
\%\end{array}$ & $\mathbf{S}_{\mathrm{AVG}}$ \\
\hline \multirow{2}{*}{1} & \multirow{2}{*}{$(\mathrm{MeCO})_{2} \mathrm{O}$} & \multirow{2}{*}{0.75 equiv } & \multirow{2}{*}{0.75} & 65.4 & 95.0 & 59.2 & 17.0 & \multirow{2}{*}{59} & \multirow{2}{*}{19} \\
\hline & & & & 69.6 & 95.0 & 57.7 & 20.0 & & \\
\hline \multirow{2}{*}{2} & \multirow{2}{*}{$(i-\mathrm{PrCO})_{2} \mathrm{O}$} & \multirow{2}{*}{0.75 equiv } & \multirow{2}{*}{48} & 86.7 & 54.6 & 38.6 & 24.2 & \multirow{2}{*}{39} & \multirow{2}{*}{26} \\
\hline & & & & 88.1 & 55.4 & 38.6 & 27.4 & & \\
\hline \multirow[b]{2}{*}{ 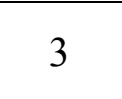 } & \multirow{2}{*}{$(\mathrm{EtCO})_{2} \mathrm{O}$} & \multirow{2}{*}{0.55 equiv } & \multirow{2}{*}{3.0} & 87.0 & 73.3 & 45.7 & 31.4 & \multirow{2}{*}{46} & \multirow{2}{*}{32} \\
\hline & & & & 86.9 & 75.0 & 46.3 & 32.0 & & \\
\hline
\end{tabular}


Procedure C2: Variation of the reaction temperature and solvent.

Stock catalyst solutions in seven different solvents containing $0.005 \mathrm{M}$ or $0.010 \mathrm{M}$ of 16 and $0.188 \mathrm{M}$ of $i-\mathrm{Pr}_{2} \mathrm{NEt}$ were prepared. Each vial charged with ( \pm )-trans-2-phenyl-cyclohexanol (44 mg, $0.25 \mathrm{mmol}$ ), $100 \mathrm{mg}$ of $\mathrm{Na}_{2} \mathrm{SO}_{4}, 1.00 \mathrm{~mL}$ of the respective stock catalyst solution and a stir bar, were stirred either at room temperature $\left(23^{\circ} \mathrm{C}\right)$ or in a cooling bath. Propionic anhydride ( $24 \mu \mathrm{L}, 0.188 \mathrm{mmol}$ ) was added to each vial. The reactions were monitored by periodically withdrawing aliquots and checking them by ${ }^{1} \mathrm{H}$ NMR. The reactions were stopped by quenching with methanol upon reaching $c a .50 \%$ conversion.

Table 4S

\begin{tabular}{|c|c|c|c|c|c|c|c|c|c|c|}
\hline Entry & $\begin{array}{c}\text { mol\% } \\
\text { of } 16\end{array}$ & solvent & $\begin{array}{l}\text { Temp } \\
\left({ }^{\circ} \mathrm{C}\right)\end{array}$ & t(h) & $\begin{array}{c}\mathbf{e e}_{\mathbf{P R}} \\
\%\end{array}$ & $\begin{array}{c}\mathbf{e e}_{\mathrm{SM}} \\
\%\end{array}$ & $\begin{array}{c}\mathrm{C}_{\mathrm{HPL}} \\
\mathrm{c} \\
\%\end{array}$ & $\mathbf{s}$ & $\begin{array}{c}\mathrm{C}_{\mathrm{AV}} \\
\mathrm{G} \\
\%\end{array}$ & $\begin{array}{c}\mathbf{S}_{\mathrm{AV}} \\
\mathrm{G}\end{array}$ \\
\hline \multirow{2}{*}{1} & \multirow{2}{*}{2} & \multirow{2}{*}{$\mathrm{CDCl}_{3}$} & \multirow{2}{*}{23} & \multirow{2}{*}{1.4} & 84.0 & 82.2 & 49.5 & 29.2 & \multirow{2}{*}{47} & \multirow{2}{*}{29} \\
\hline & & & & & 86.9 & 69.4 & 44.4 & 29.6 & & \\
\hline \multirow{2}{*}{2} & \multirow{2}{*}{2} & \multirow{2}{*}{$\mathrm{CDCl}_{3}$} & \multirow{2}{*}{0} & \multirow{2}{*}{2} & 85.9 & 92.4 & 51.8 & 43.5 & \multirow{2}{*}{52} & \multirow{2}{*}{43} \\
\hline & & & & & 84.5 & 94.6 & 52.8 & 42.8 & & \\
\hline \multirow{2}{*}{3} & \multirow{2}{*}{2} & \multirow{2}{*}{$\mathrm{CDCl}_{3}$} & \multirow{2}{*}{-20} & \multirow{2}{*}{3} & 83.7 & 98.5 & 54.1 & 54.2 & \multirow{2}{*}{53} & \multirow{2}{*}{53} \\
\hline & & & & & 86.7 & 95.1 & 52.3 & 52.1 & & \\
\hline \multirow{3}{*}{4} & \multirow{3}{*}{4} & \multirow{3}{*}{$\mathrm{CDCl}_{3}$} & \multirow{3}{*}{-40} & \multirow{3}{*}{4} & 88.8 & 99.2 & 52.8 & 91.4 & \multirow{3}{*}{53} & \multirow{3}{*}{86} \\
\hline & & & & & 87.9 & 98.9 & 53.0 & 79.8 & & \\
\hline & & & & & 88.4 & 99.1 & 52.9 & 86.0 & & \\
\hline \multirow{2}{*}{5} & \multirow{2}{*}{4} & \multirow{2}{*}{$\mathrm{CDCl}_{3}$} & 55 & & 95.1 & 89.2 & 48.4 & 119.4 & & \\
\hline & & & -52 & 4 & 96.4 & 75.7 & 44.0 & 124.3 & 46 & 122 \\
\hline & & & & & 75.3 & 68.8 & 47.8 & 14.5 & & \\
\hline 6 & 2 & MecN & 23 & 2.5 & 75.6 & 69.8 & 48.0 & 14.9 & 48 & 15 \\
\hline 7 & 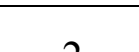 & $\mathrm{CH} \mathrm{Cl}$ & 2 & 10 & 82.4 & 73.6 & 47.2 & 22.7 & 17 & 20 \\
\hline t & 2 & $\mathrm{CH}_{2} \mathrm{Cl}_{2}$ & 23 & 1.8 & 83.6 & 71.4 & 46.1 & 23.7 & 4/ & 23 \\
\hline 0 & 2 & TUF & 23 & 18 & 81.9 & 81.1 & 49.8 & 25.0 & 50 & 25 \\
\hline 8 & 2 & $1 \Pi \mathrm{F}$ & 23 & 1.8 & 82.3 & 81.3 & 49.7 & 25.7 & 50 & 20 \\
\hline 9 & 2 & $\mathrm{PhMe}$ & 23 & 1 & 80.6 & 92.6 & 53.5 & 30.7 & 54 & 30 \\
\hline 9 & 2 & Pnivie & 23 & 1 & 78.1 & 95.4 & 55.0 & 30.2 & 34 & 30 \\
\hline 10 & 2 & $\mathrm{TA}^{\mathrm{a}}$ & 23 & 07 & 79.3 & 98.5 & 55.4 & 40.9 & 56 & 41 \\
\hline 10 & 2 & & & & 77.8 & 98.9 & 56.0 & 40.0 & & \\
\hline 12 & 2 & $T A^{a}$ & -10 & 2 & 85.4 & 99.3 & 53.8 & 70.0 & 54 & 72 \\
\hline & & & & & 85.5 & 99.5 & 538 & 74.7 & 34 & 12 \\
\hline 13 & 4 & $1: 1$ & -40 & 3 & 94.9 & 83.6 & 46.8 & 100.4 & 46 & 101 \\
\hline & & $\mathrm{TA}^{\mathrm{a}} / \mathrm{PhMe}$ & & & 95.4 & 78.2 & 45.1 & 101.7 & 40 & \\
\hline
\end{tabular}

$\left.{ }^{\mathrm{a}}\right) \mathrm{TA}=$ tert-amyl alcohol

Procedure D1: KR catalyzed by HBTM 16 at room temperature in $\mathrm{CDCl}_{3}$.

The stock solution of the catalyst was prepared by dissolving 0.050 mmol catalyst 16 and $238 \mu \mathrm{L}$ $\left(1.38 \mathrm{mmol}\right.$ ) of $i-\mathrm{Pr}_{2} \mathrm{NEt}$ in $\mathrm{CDCl}_{3}$ in a $10.00 \mathrm{~mL}$ volumetric test tube and bringing the volume to the mark. A one dram vial was charged with $0.25 \mathrm{mmol}$ of substrates and $1.00 \mathrm{~mL}$ of the stock 
catalyst solution. Propionic anhydride $(17.6 \mu \mathrm{L}, 0.138 \mathrm{mmol})$ was added. The reaction was monitored by ${ }^{1} \mathrm{H}$ NMR at room temperature and stopped by adding several drops of $\mathrm{MeOH}$ upon reaching ca. $50 \%$ conversion.

Table 5S.

\begin{tabular}{|c|c|c|c|c|c|c|c|c|}
\hline Entry & Substrate & $\mathbf{t} \mathbf{( h )}$ & $\begin{array}{c}\mathbf{e e}_{\mathbf{P R}} \\
\mathbf{\%}\end{array}$ & $\begin{array}{c}\mathbf{e e}_{\text {SM }} \\
\mathbf{\%}\end{array}$ & $\begin{array}{c}\mathbf{C}_{\mathbf{H P L C}} \\
\mathbf{\%}\end{array}$ & $\mathbf{s}$ & $\begin{array}{c}\mathbf{C}_{\text {AVG }} \\
\mathbf{\%}\end{array}$ & $\mathbf{S}_{\text {AVG }}$ \\
\hline 1 & $\mathbf{2 6}$ & 1.7 & 80.0 & 58.5 & 42.3 & 16.2 & 42 & 16 \\
\hline $2^{\text {a }}$ & $\mathbf{2 7}$ & 26 & 92.4 & 64.5 & 41.1 & 49.2 & 41 & 49 \\
\hline 3 & $\mathbf{2 8}$ & 1.2 & 72.3 & 98.1 & 57.6 & 27.7 & 58 & 28 \\
\hline 4 & $\mathbf{2 9}$ & 24 & 80.9 & 46.4 & 36.5 & 14.9 & 37 & 15 \\
\hline 5 & $\mathbf{3 0}$ & 1.8 & 49.5 & 49.3 & 49.9 & 4.7 & 50 & 4.7 \\
\hline 6 & $\mathbf{3 1}$ & 12 & 59.2 & 38.1 & 39.1 & 5.6 & 39 & 5.6 \\
\hline 7 & $\mathbf{3 2}$ & 1.3 & 62.7 & 58.6 & 48.3 & 7.8 & 48 & 7.8 \\
\hline 8 & $\mathbf{3 3}$ & 22 & 30.6 & 20.6 & 40.3 & 2.3 & 40 & 2.3 \\
\hline 9 & $\mathbf{3 4}$ & 1.3 & 55.9 & 48.9 & 46.7 & 5.6 & 47 & 5.6 \\
\hline 10 & $\mathbf{2 2}$ & 1 & 83.3 & 79.2 & 48.8 & 26.4 & 49 & 26 \\
\hline 11 & $\mathbf{3 5}$ & 0.4 & 83.4 & 76.0 & 47.7 & 25.1 & 48 & 25 \\
\hline
\end{tabular}

$\left.{ }^{\mathrm{a}}\right)$ The concentration was $0.1 \mathrm{M}$ instead of 0.25 due to poor solubility.

\section{Procedure D2: KR catalyzed by HBTM 16 at $-10{ }^{\circ} \mathrm{C}$ in $t$-amyl alcohol.}

The stock solution of the catalyst was prepared by dissolving $0.050 \mathrm{mmol}$ catalyst 16 and $325 \mu \mathrm{L}$ $(1.88 \mathrm{mmol})$ of $i-\mathrm{Pr}_{2} \mathrm{NEt}$ in $t$-amyl alcohol in a $10.00 \mathrm{~mL}$ volumetric test tube and bringing the volume to the mark. Each KR experiment was performed in duplicate. A one dram vial was charged with $0.25 \mathrm{mmol}$ of a substrate and $1.00 \mathrm{~mL}$ of the stock solution of the catalyst and stirred at $-10{ }^{\circ} \mathrm{C}$ for 15 min followed by addition of propionic anhydride $(24 \mu \mathrm{L}, 0.188 \mathrm{mmol})$. The reaction was stopped after $12 \mathrm{~h}$ by adding $\mathrm{MeOH}$.

Table 6S.

\begin{tabular}{|c|c|c|c|c|c|c|c|c|}
\hline Entry & Substrate & t (h) & $\begin{array}{c}\mathbf{e e}_{\mathrm{PR}} \\
\% \\
\end{array}$ & $\begin{array}{c}\mathbf{e e}_{\mathrm{SM}} \\
\% \\
\end{array}$ & $\begin{array}{c}\mathbf{C}_{\text {HPLC }} \\
\% \\
\end{array}$ & $\mathbf{S}$ & $\begin{array}{c}\mathrm{C}_{\mathrm{AVG}} \\
\% \\
\end{array}$ & $\mathbf{S}_{\mathrm{AVG}}$ \\
\hline \multirow{2}{*}{1} & \multirow{2}{*}{29} & \multirow{2}{*}{12} & 85.9 & 72.2 & 45.7 & 28.3 & \multirow{2}{*}{46} & \multirow{2}{*}{28} \\
\hline & & & 85.9 & 72.2 & 45.7 & 28.3 & & \\
\hline \multirow{2}{*}{2} & \multirow{2}{*}{31} & \multirow{2}{*}{12} & 61.2 & 79.1 & 56.4 & 9.7 & \multirow{2}{*}{55} & \multirow{2}{*}{10} \\
\hline & & & 64.3 & 75.9 & 54.1 & 10.3 & & \\
\hline \multirow[t]{2}{*}{3} & \multirow{2}{*}{33} & \multirow{2}{*}{10} & 45.2 & 21.3 & 32.0 & 3.2 & \multirow{2}{*}{33} & \multirow{2}{*}{3.2} \\
\hline & & & 44.6 & 22.8 & 33.8 & 3.2 & & \\
\hline
\end{tabular}

Procedure D3: KR catalyzed by HBTM 16 at $-40{ }^{\circ} \mathrm{C}$ in $t$-amyl alcohol-toluene mixture.

The stock solution of the catalyst was prepared by dissolving $0.050 \mathrm{mmol}$ catalyst 16 and $325 \mu \mathrm{L}$ $(1.88 \mathrm{mmol})$ of $i$ - $\mathrm{Pr}_{2} \mathrm{NEt}$ in a $t$-amyl alcohol-toluene mixture $(1: 1 \mathrm{vol} / \mathrm{vol})$ in a $10.00 \mathrm{~mL}$ volumetric test tube and bringing the volume to the mark. Each KR experiment was performed in duplicate. A one-dram vial was charged with $0.25 \mathrm{mmol}$ of a substrate and $1.00 \mathrm{~mL}$ of the stock 
catalyst solution and stirred at $-40{ }^{\circ} \mathrm{C}$ for 15 min followed by addition of propionic anhydride $(24$ $\mu \mathrm{L}, 0.188 \mathrm{mmol})$. All reaction mixtures were stopped after $10 \mathrm{~h}$ except for those with substrates 28 and 35, which were quenched after $7 \mathrm{~h}$ and $5 \mathrm{~h}$, respectively.

Table 7S

\begin{tabular}{|c|c|c|c|c|c|c|c|c|}
\hline Entry & Substrate & t (h) & $\begin{array}{c}\mathbf{e e}_{\mathrm{PR}} \\
\%\end{array}$ & $\begin{array}{c}\mathbf{e e}_{\mathrm{SM}} \\
\%\end{array}$ & $\begin{array}{c}\mathrm{C}_{\mathrm{HPLC}} \\
\%\end{array}$ & $\mathbf{s}$ & $\begin{array}{c}\mathrm{C}_{\mathrm{AVG}} \\
\%\end{array}$ & $\mathbf{s}_{\mathrm{AVG}}$ \\
\hline \multirow[b]{2}{*}{1} & \multirow[b]{2}{*}{24} & \multirow[b]{2}{*}{10} & 93.2 & 95.5 & 50.6 & 109.0 & \multirow[b]{2}{*}{51} & \multirow[b]{2}{*}{107} \\
\hline & & & 93.2 & 94.9 & 50.5 & 105.4 & & \\
\hline \multirow[b]{2}{*}{2} & \multirow[b]{2}{*}{26} & \multirow[b]{2}{*}{10} & 90.4 & 73.4 & 44.8 & 43.5 & \multirow[b]{2}{*}{44} & \multirow{2}{*}{44} \\
\hline & & & 91.4 & 67.6 & 42.5 & 45.0 & & \\
\hline \multirow{2}{*}{3} & \multirow{2}{*}{28} & \multirow{2}{*}{7} & 89.5 & 94.2 & 51.3 & 64.6 & \multirow{2}{*}{51} & \multirow{2}{*}{66} \\
\hline & & & 89.4 & 95.2 & 51.6 & 67.2 & & \\
\hline \multirow{2}{*}{4} & \multirow{2}{*}{30} & \multirow{2}{*}{10} & 63.1 & 27.9 & 30.7 & 5.8 & \multirow{2}{*}{28} & \multirow{2}{*}{5.6} \\
\hline & & & 62.9 & 22.2 & 26.1 & 5.4 & & \\
\hline \multirow{2}{*}{5} & \multirow{2}{*}{32} & \multirow{2}{*}{10} & 76.8 & 25.7 & 25.1 & 9.8 & \multirow{2}{*}{26} & \multirow{2}{*}{10} \\
\hline & & & 77.4 & 27.1 & 25.9 & 10.2 & & \\
\hline \multirow{2}{*}{6} & \multirow{2}{*}{34} & \multirow{2}{*}{10} & 70.9 & 33.9 & 32.3 & 8.1 & \multirow{2}{*}{34} & \multirow{2}{*}{7.9} \\
\hline & & & 68.5 & 37.7 & 35.5 & 7.7 & & \\
\hline \multirow{2}{*}{7} & \multirow{2}{*}{22} & \multirow{2}{*}{10} & 93.1 & 60.8 & 39.5 & 52.3 & \multirow{2}{*}{39} & \multirow{2}{*}{55} \\
\hline & & & 94.1 & 56.9 & 37.7 & 58.3 & & \\
\hline \multirow{2}{*}{8} & 35 & 5 & 93.1 & 55.0 & 37.2 & 48.7 & 37 & 10 \\
\hline & 30 & $J$ & 93.3 & 54.4 & 36.9 & 49.7 & 37 & 49 \\
\hline
\end{tabular}




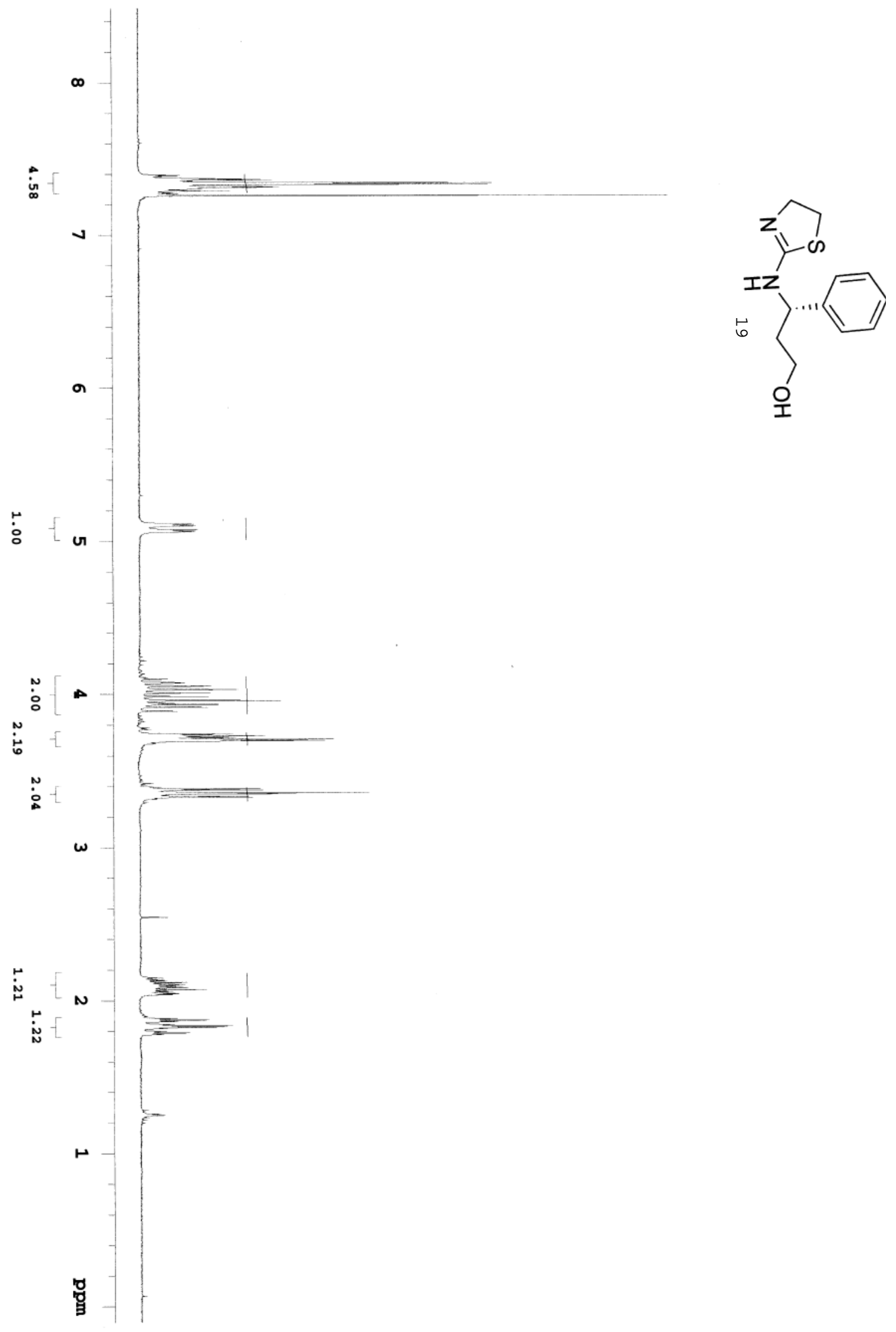

S-13 


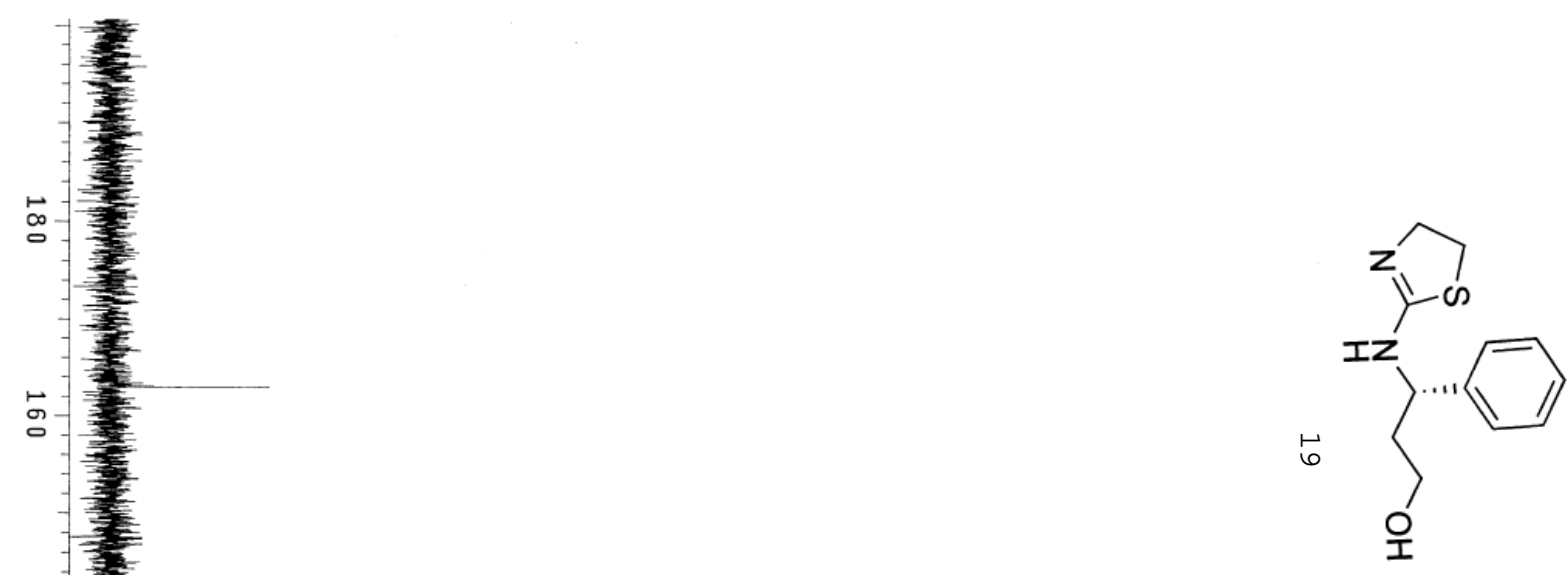

吕

․-

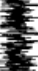

$\stackrel{\infty}{\circ}$

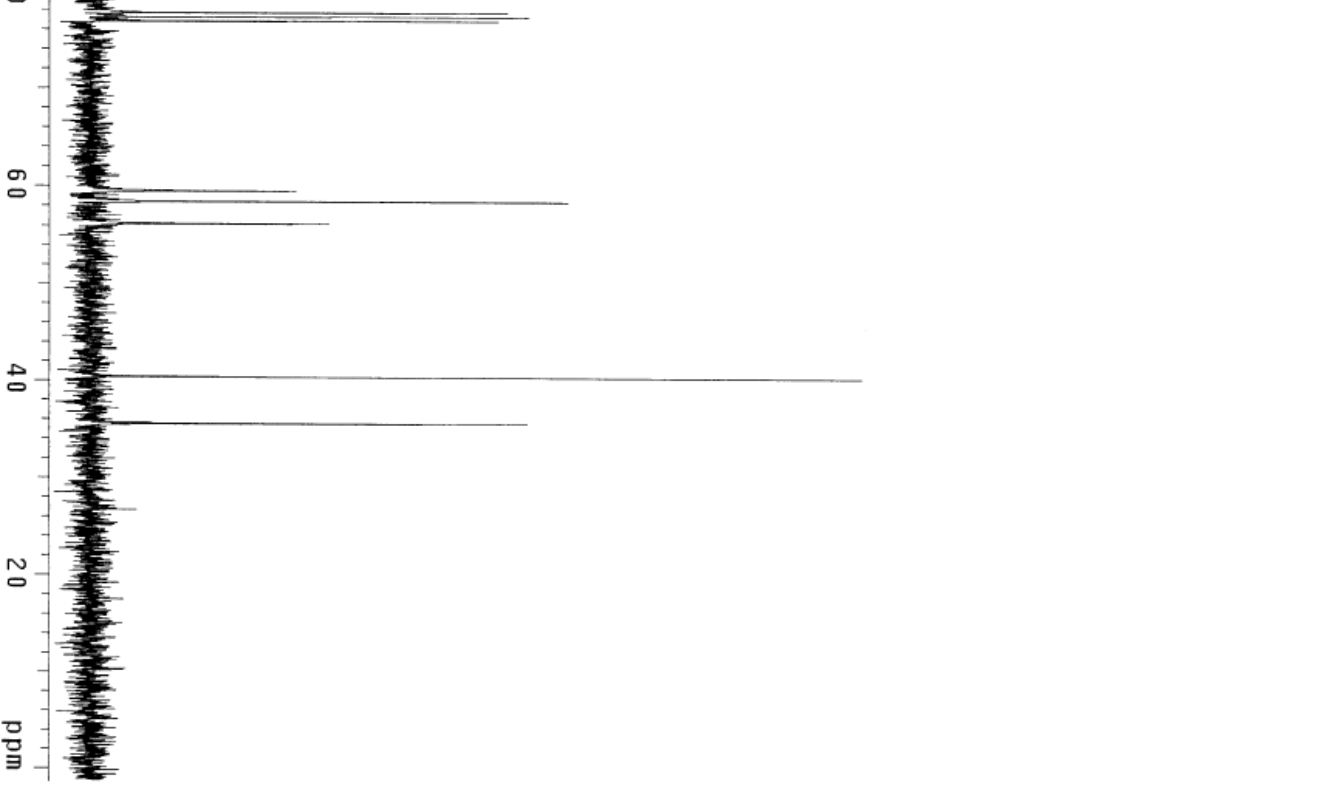



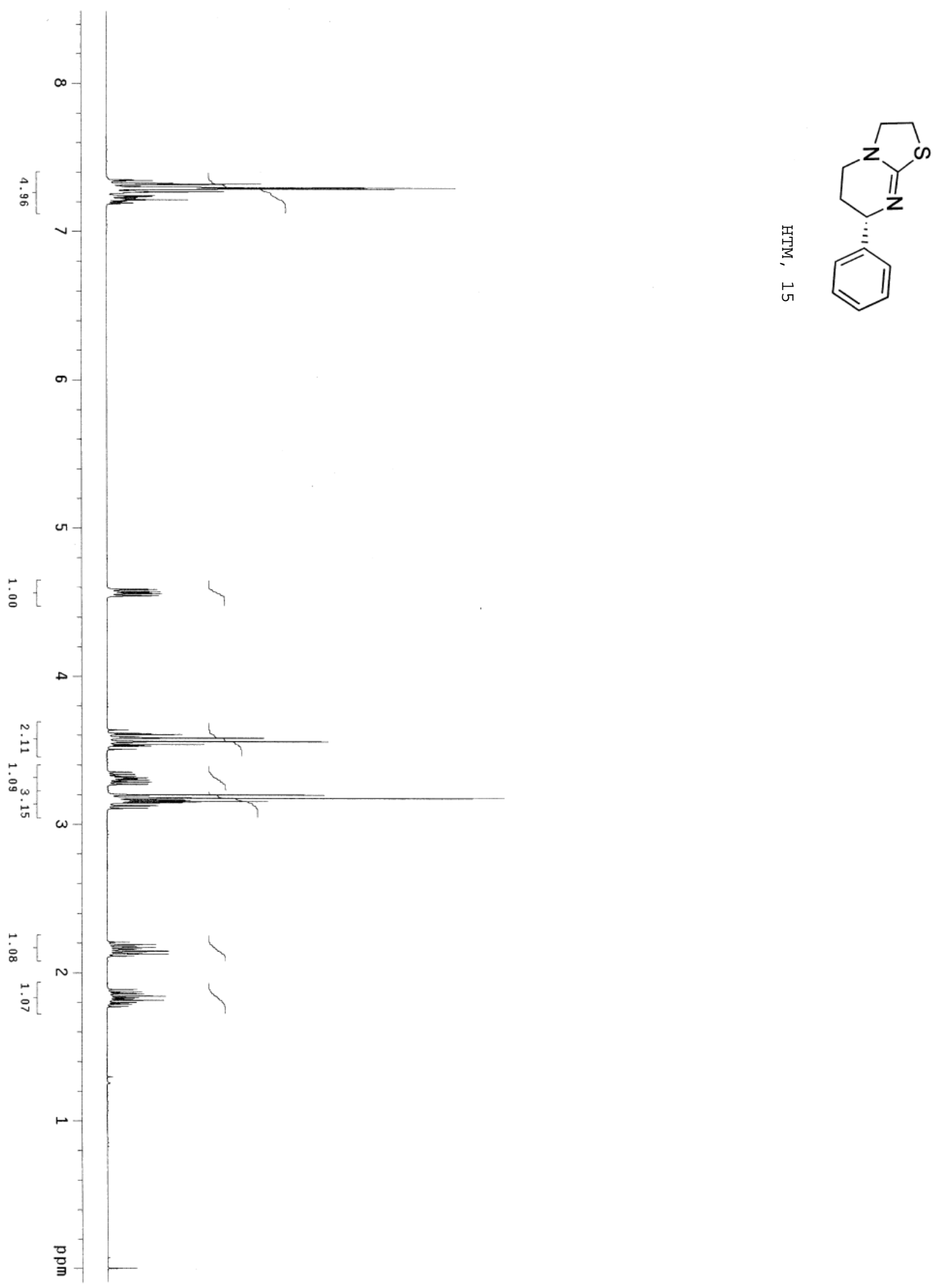

S-15 


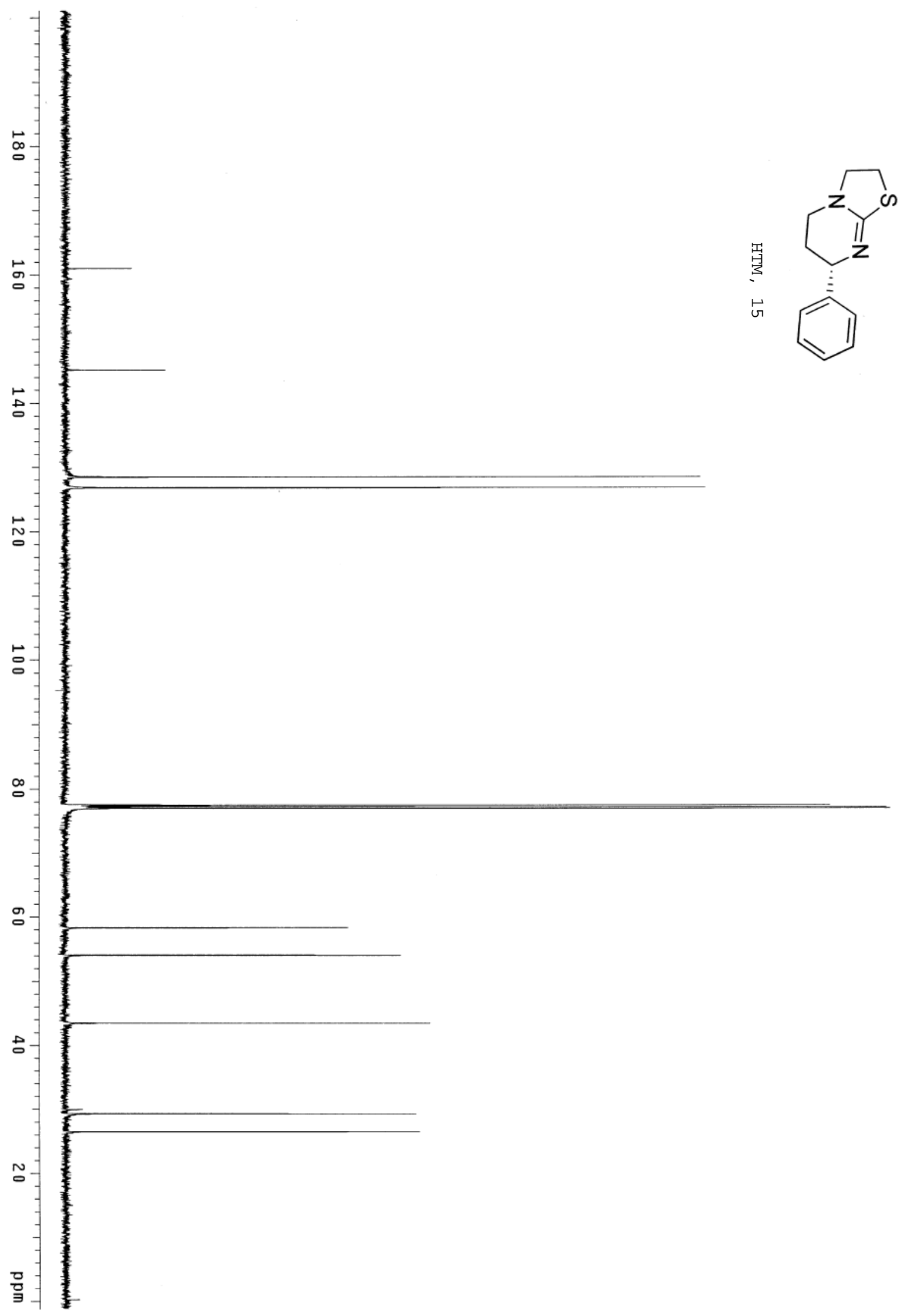

S-16 

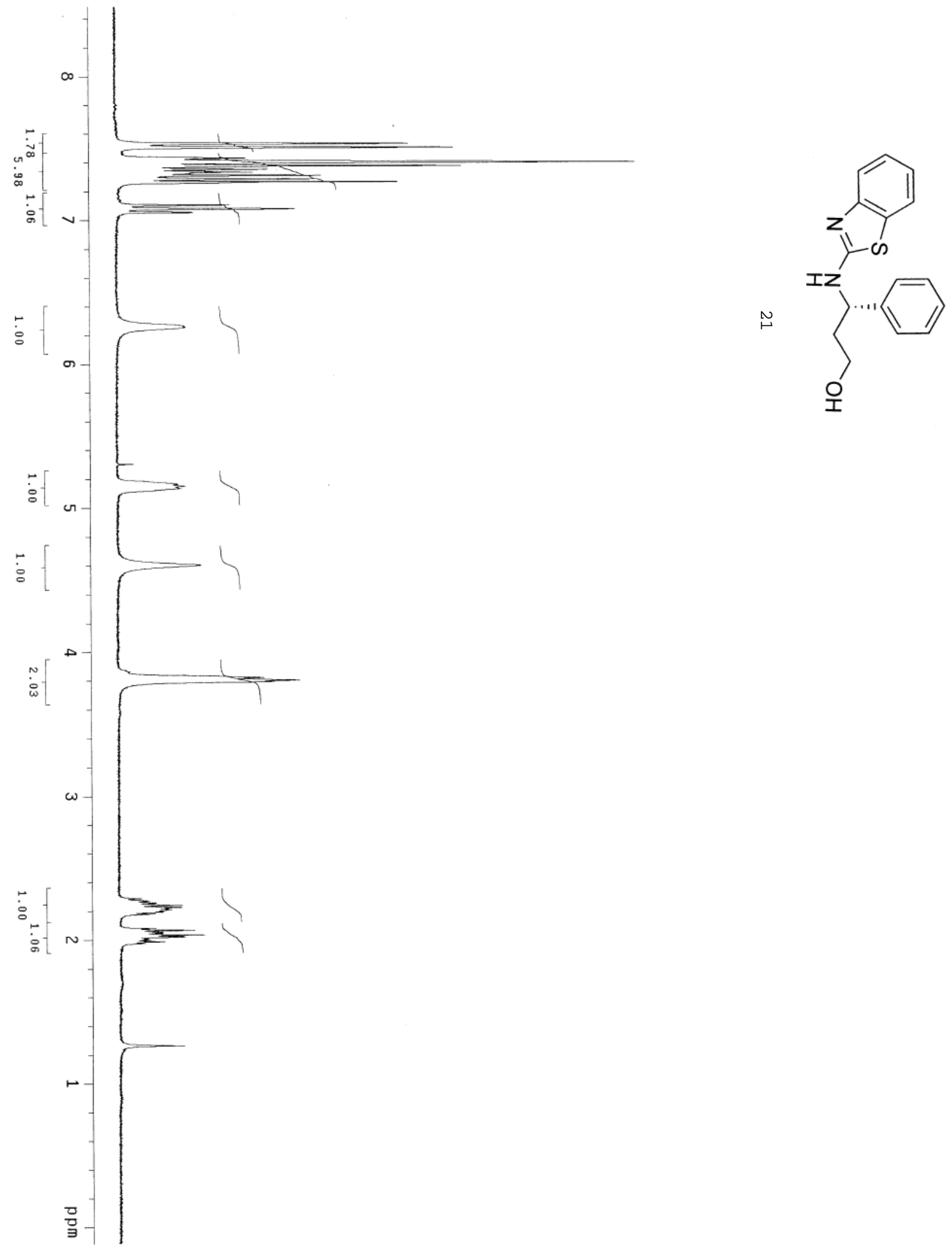

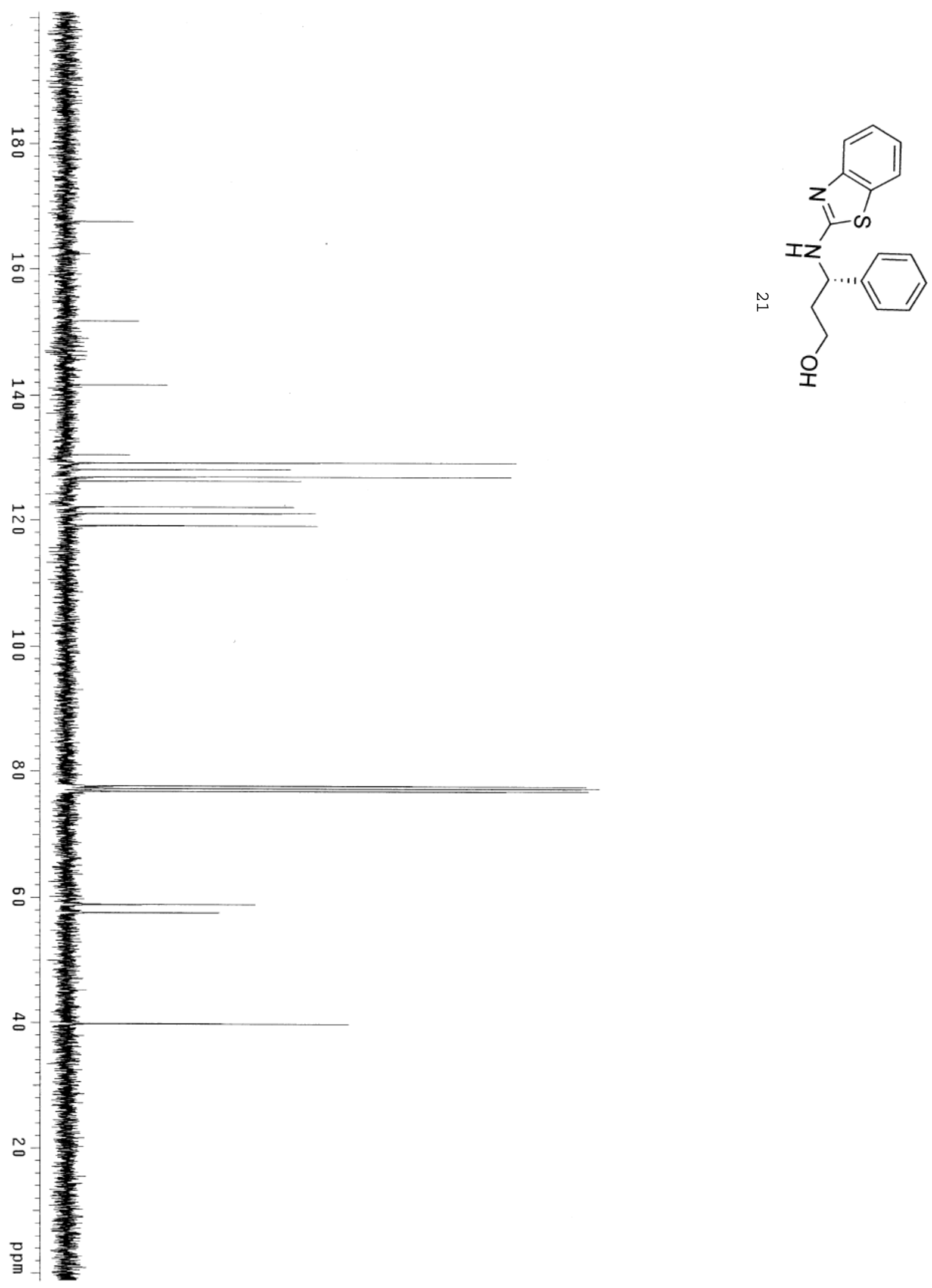

S-18 


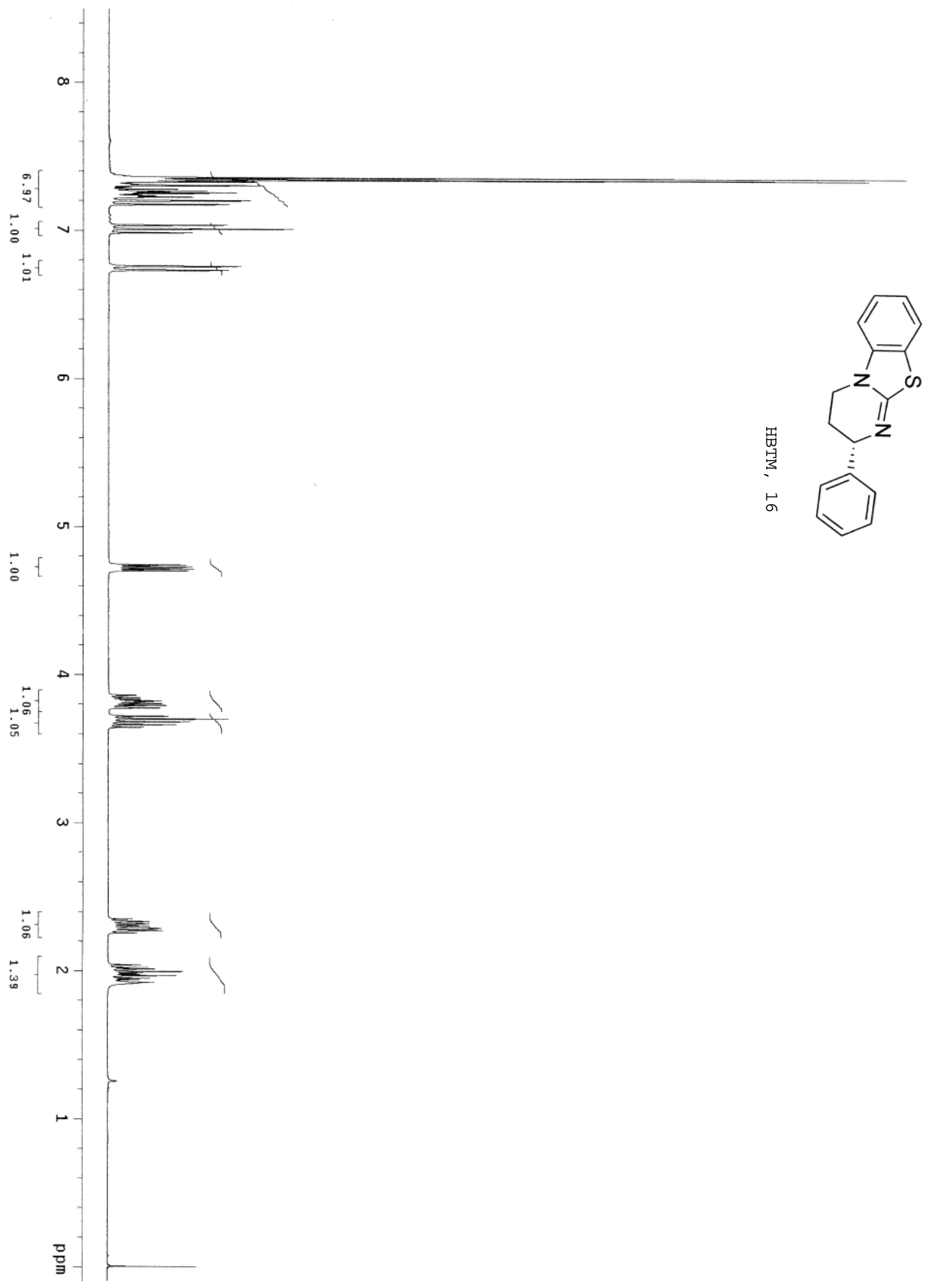

S-19 

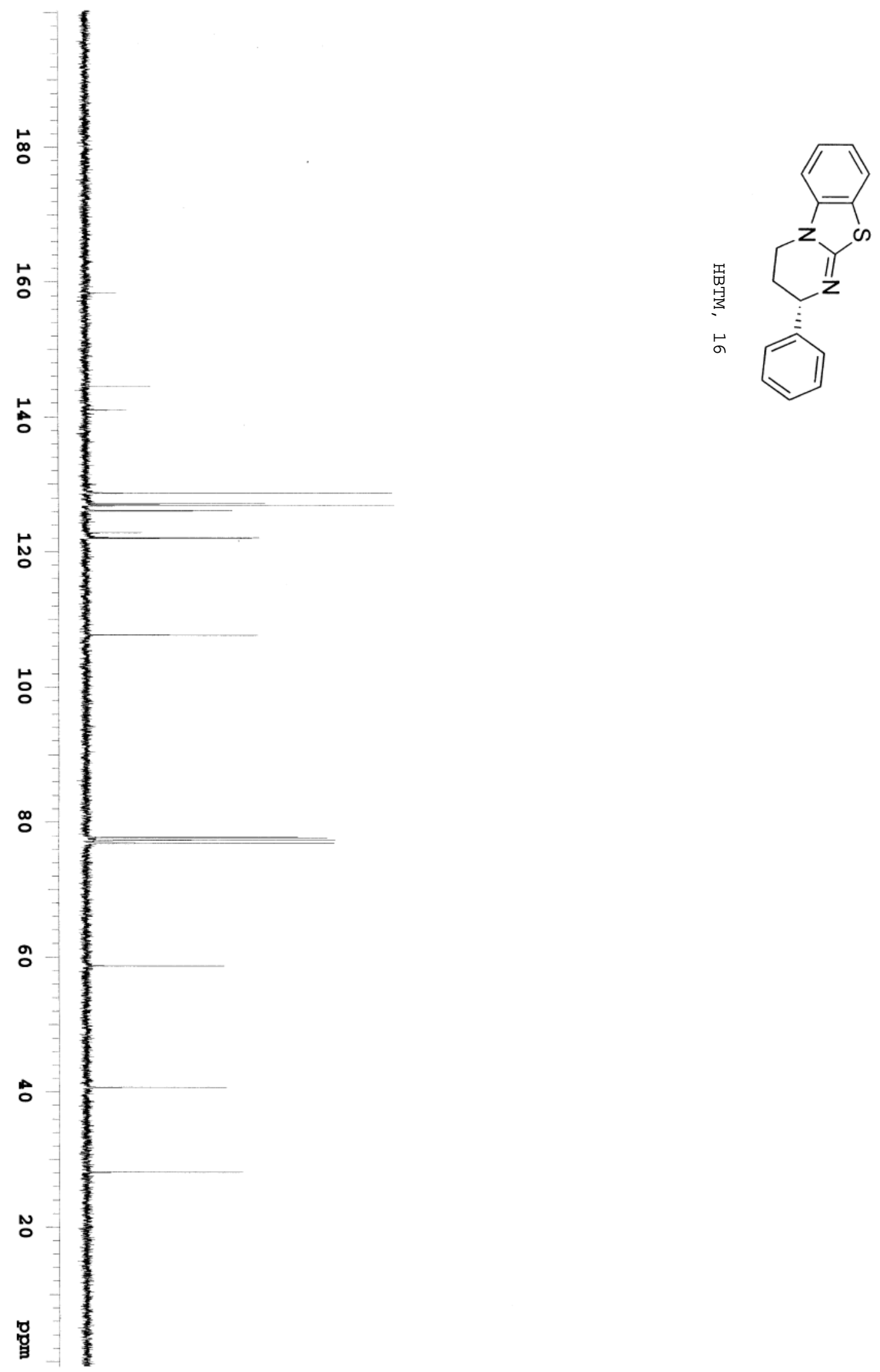

S-20 

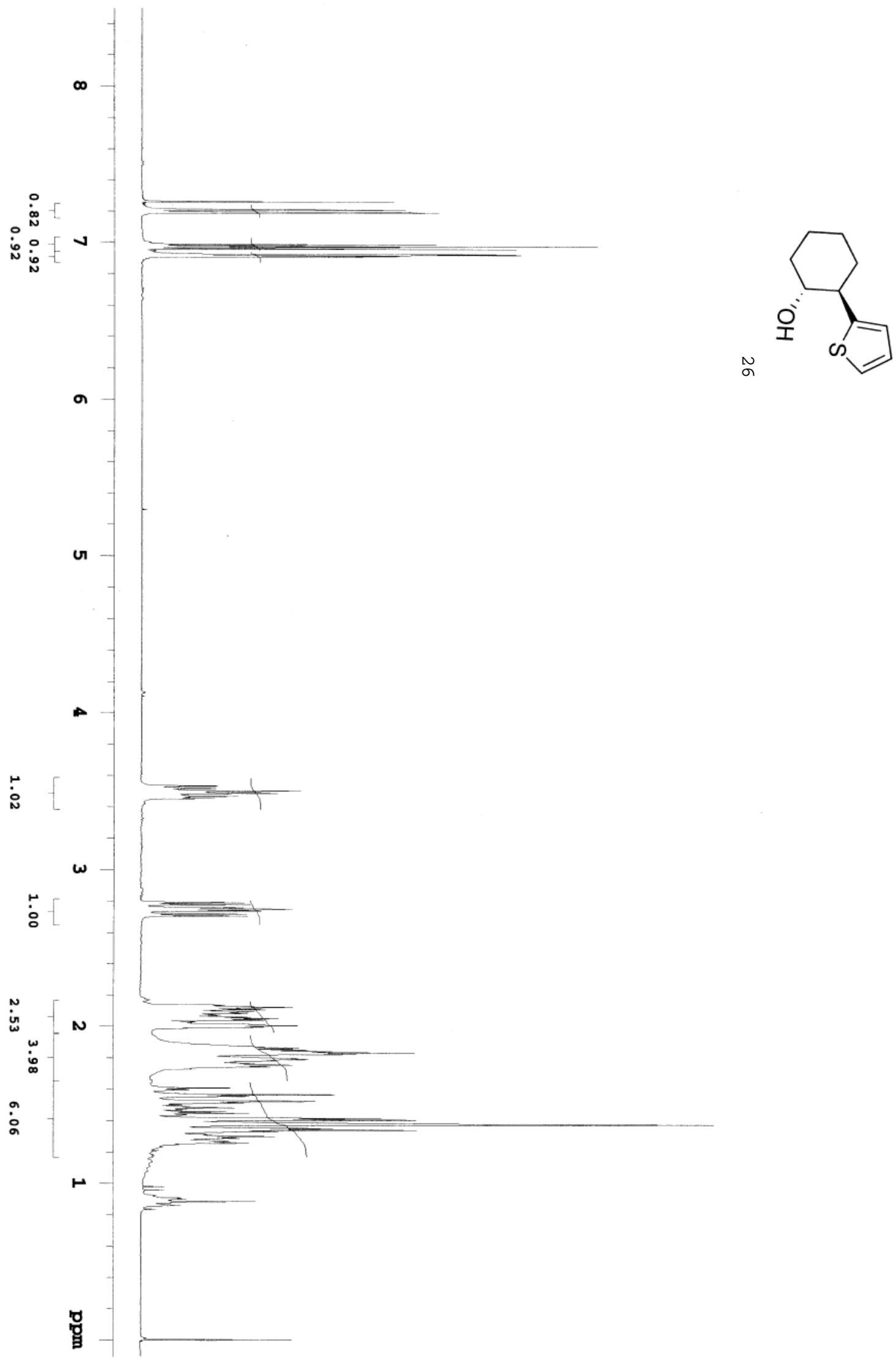


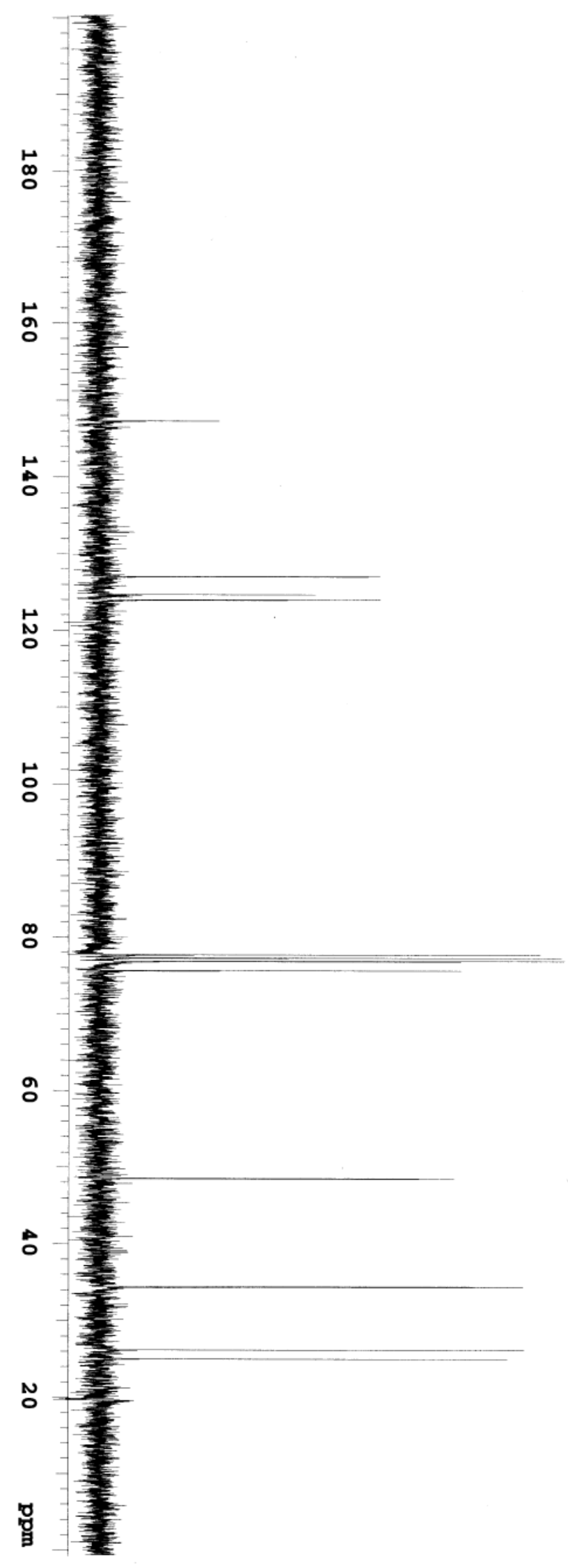




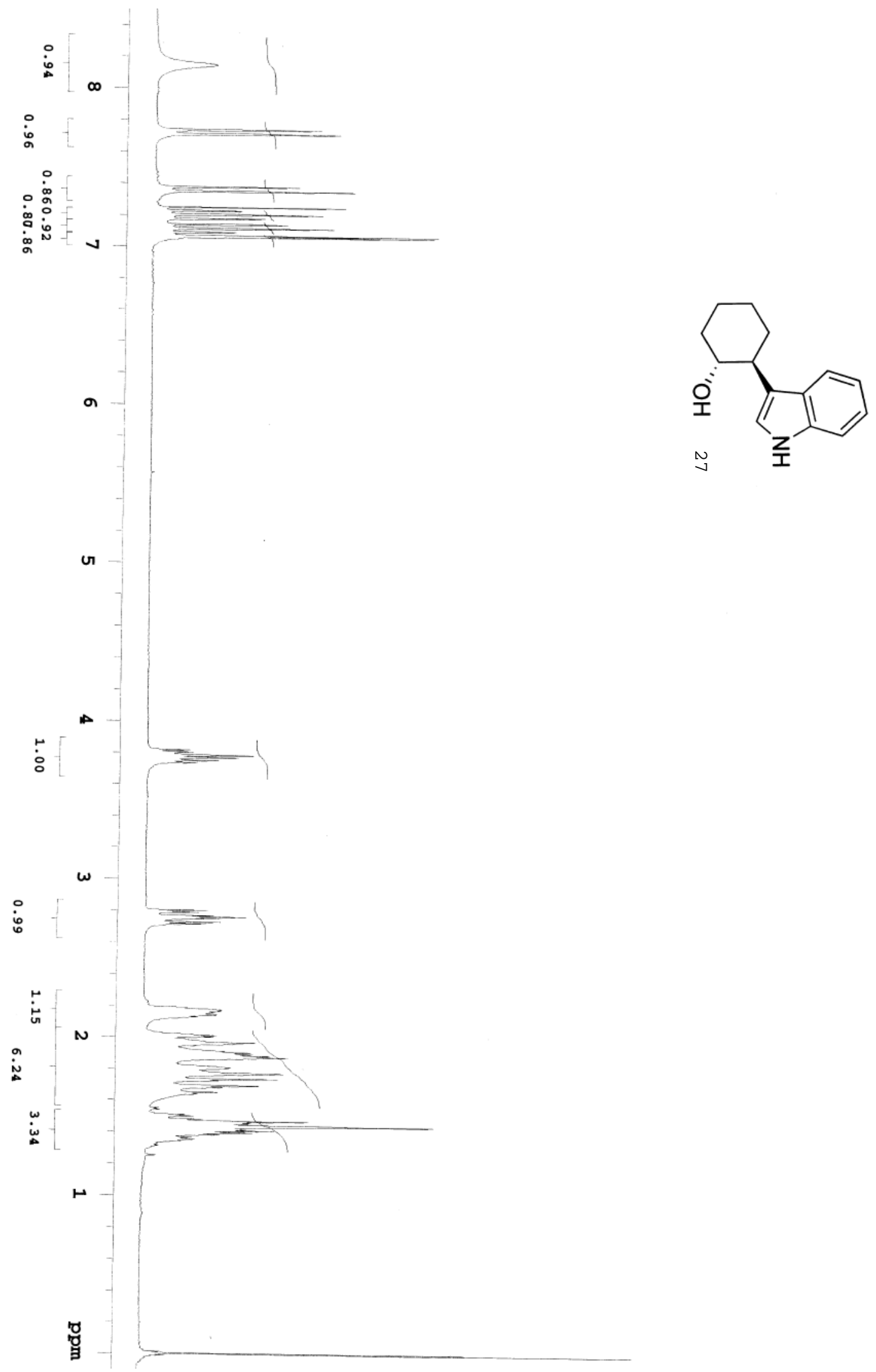

S-23 

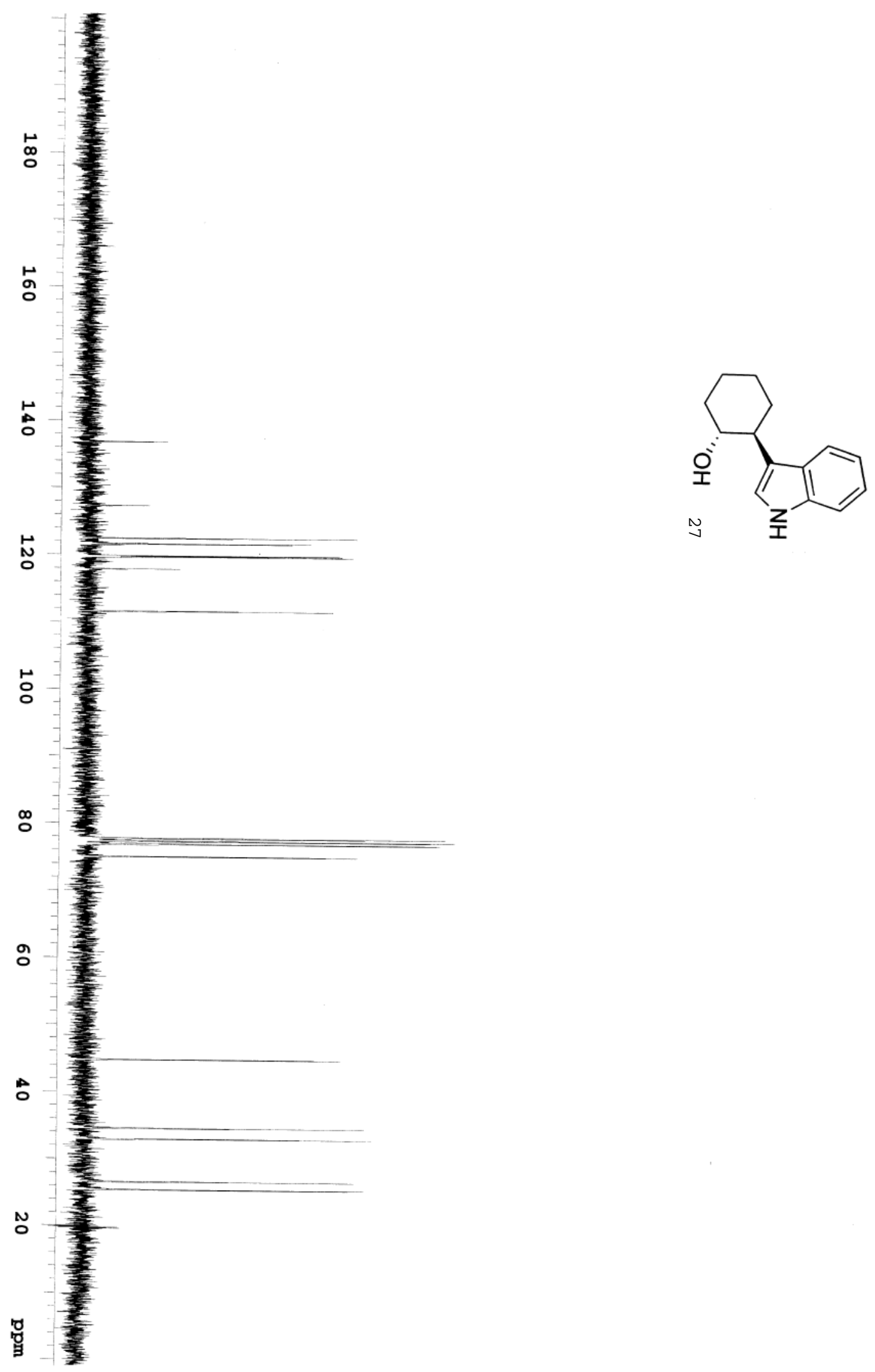

S-24 


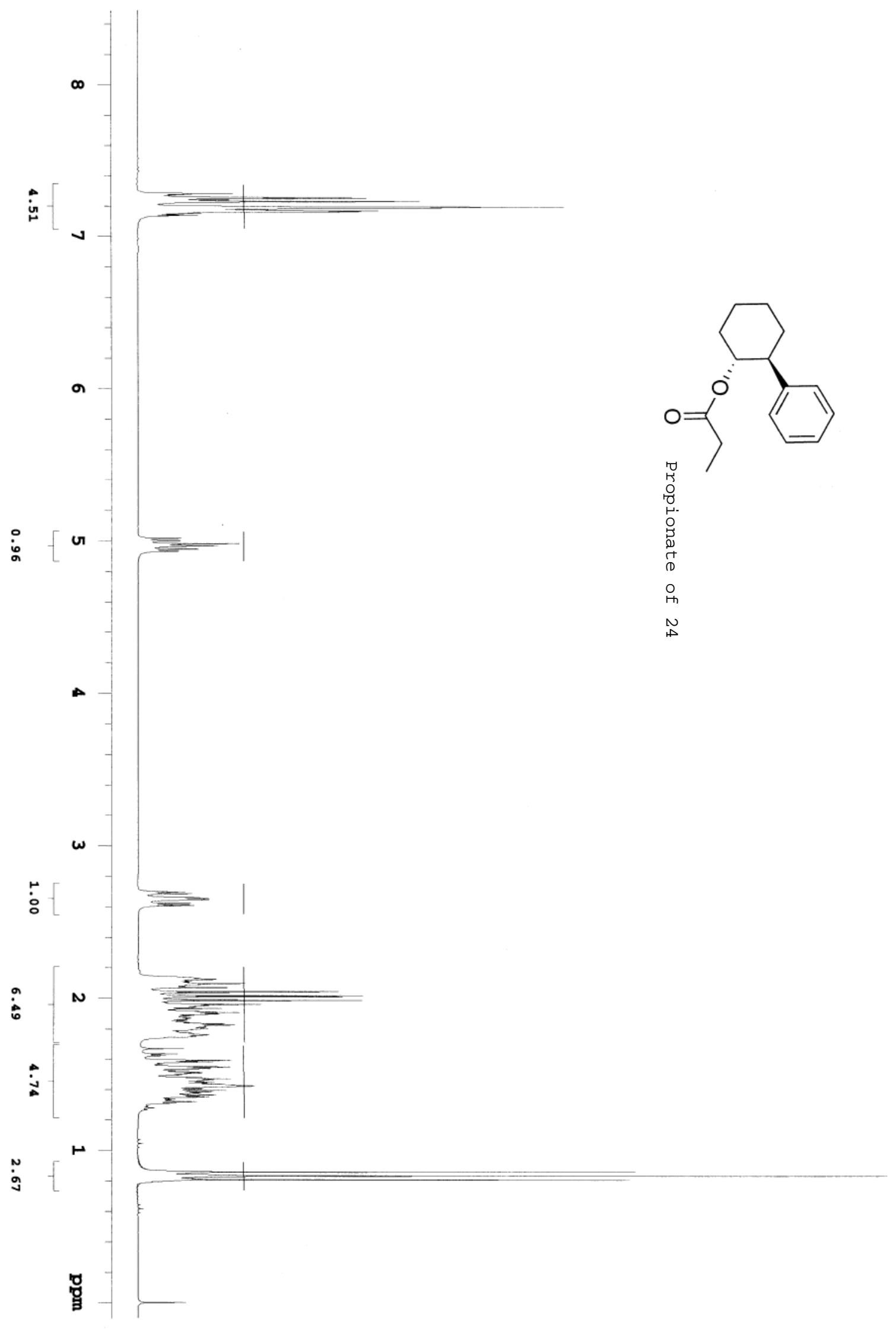

S-25 

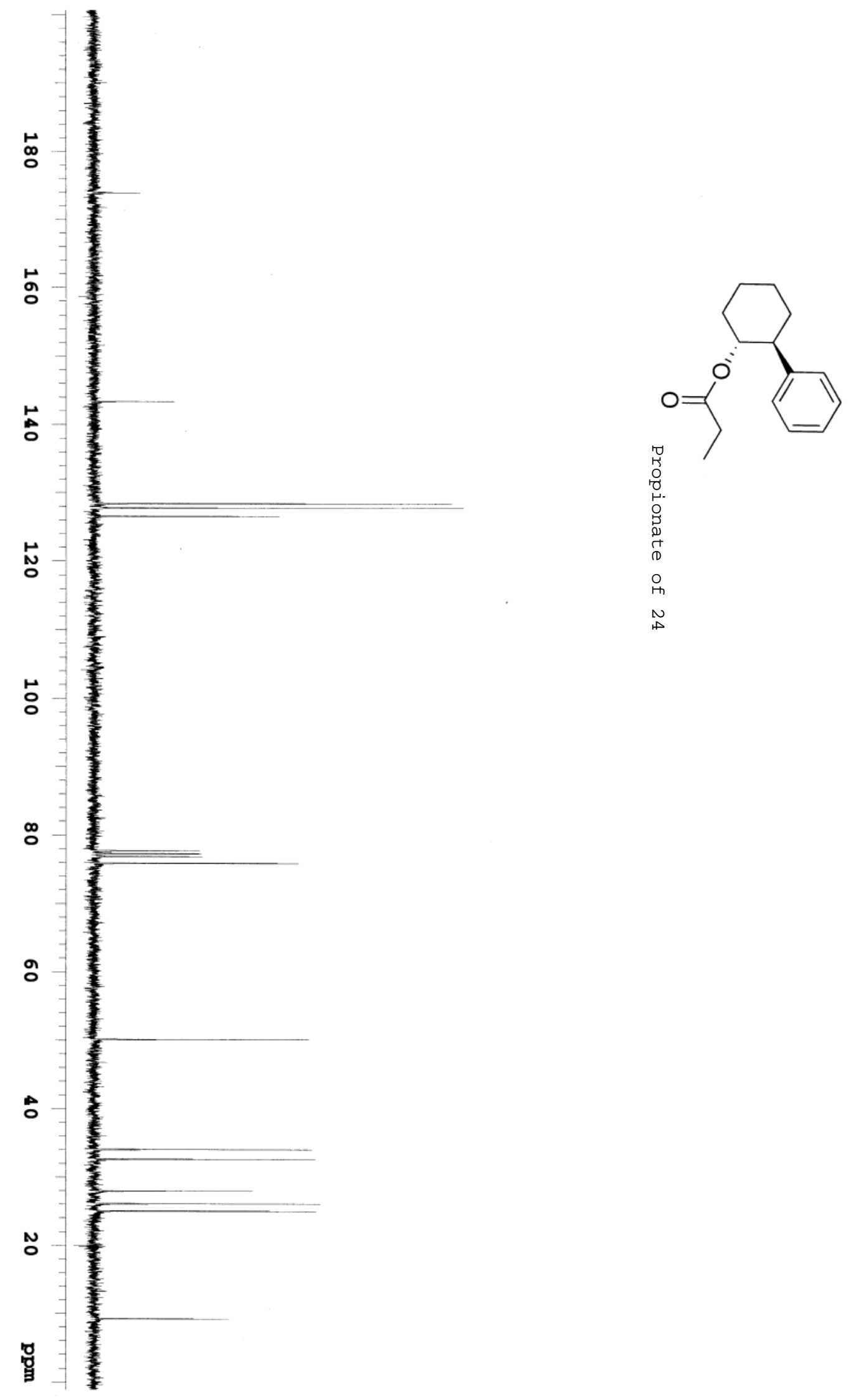

S-26 


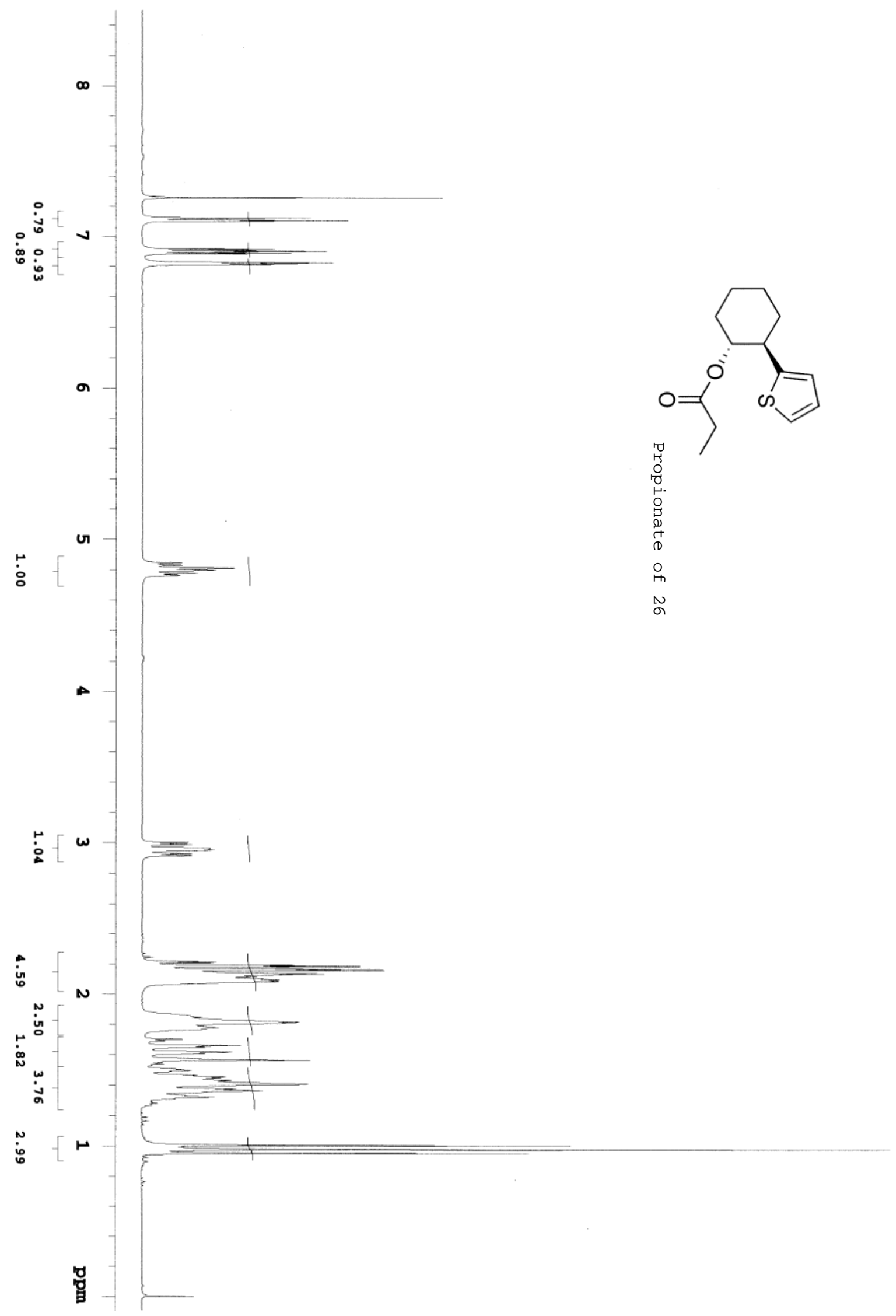

S-27 

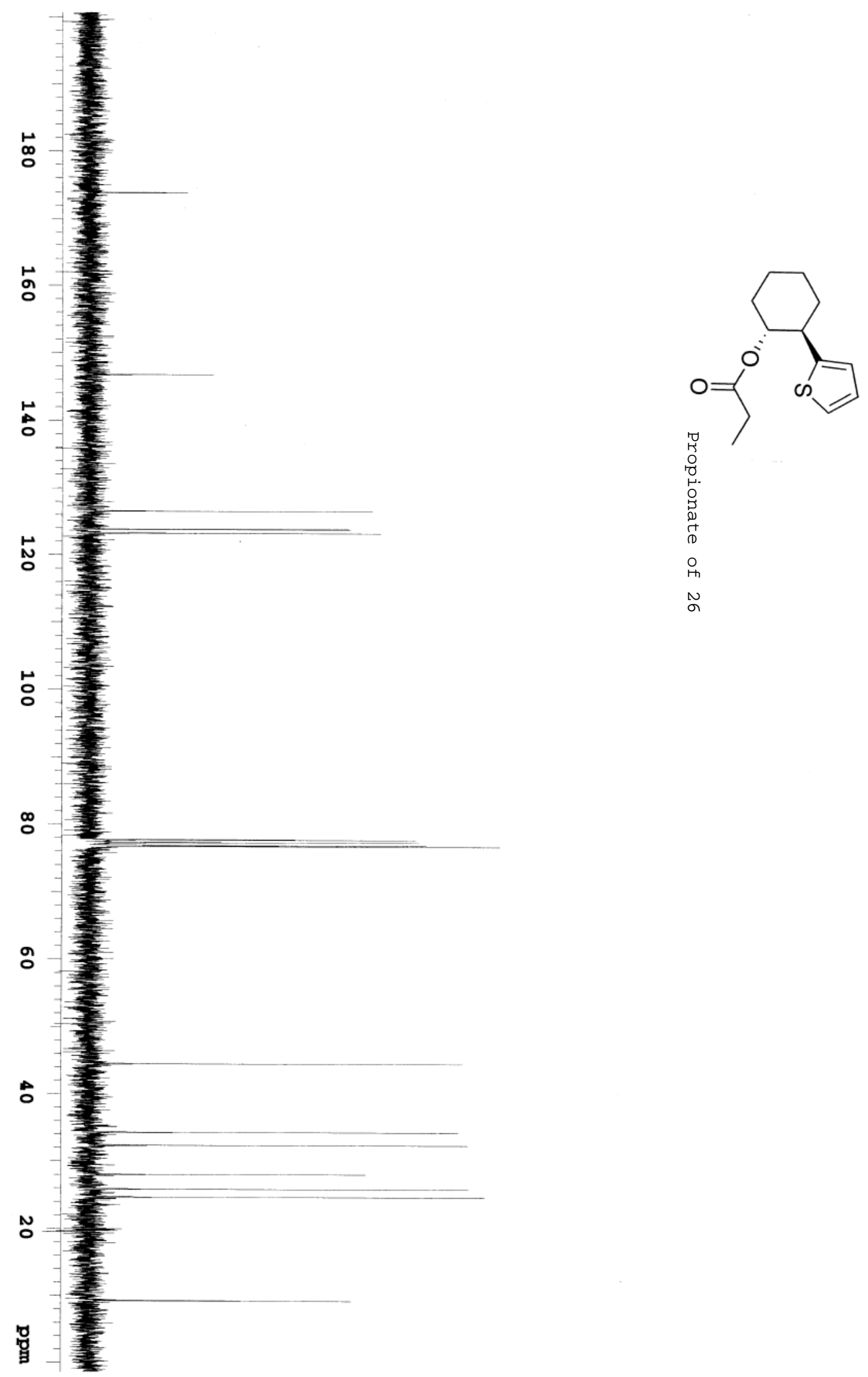

S-28 


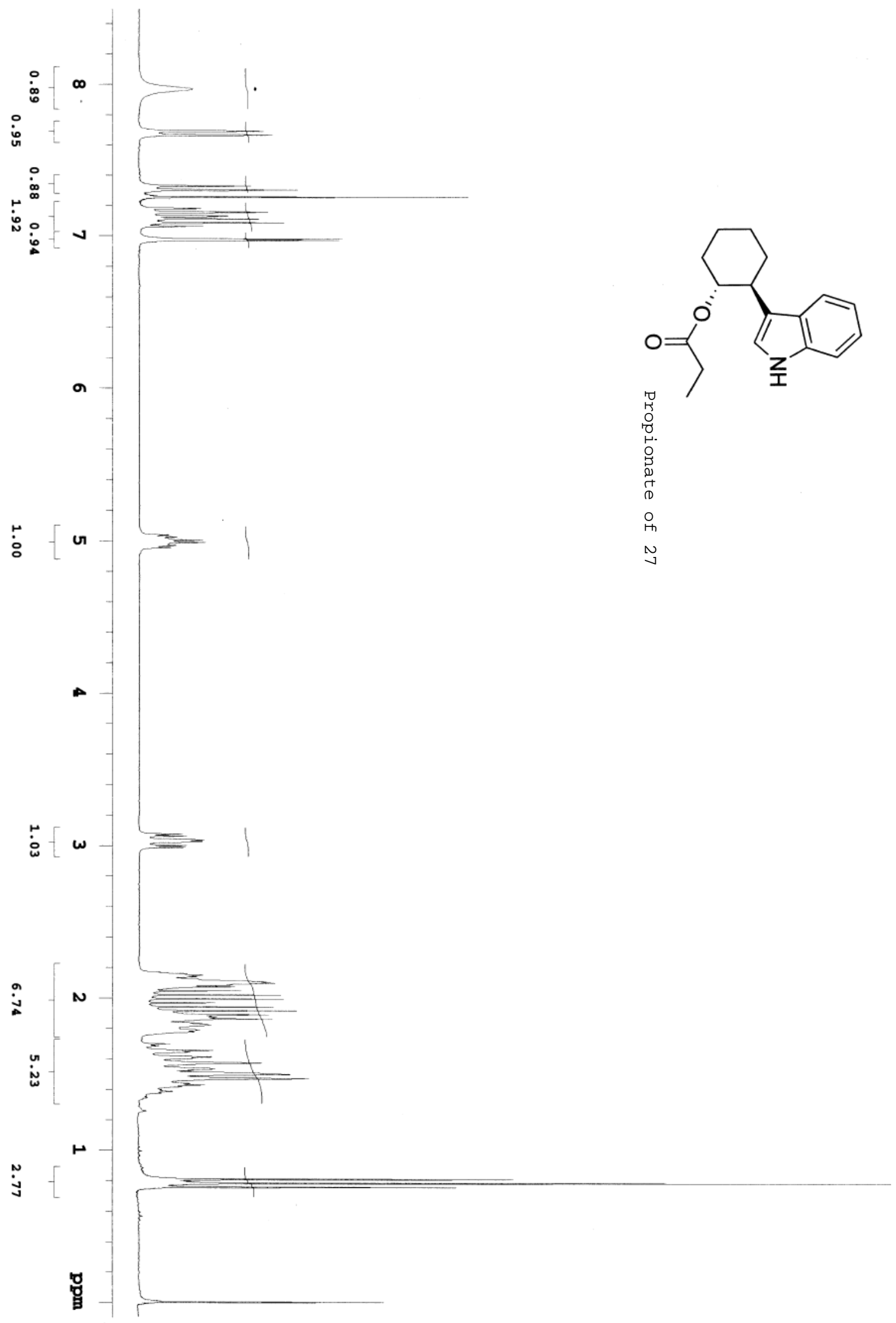




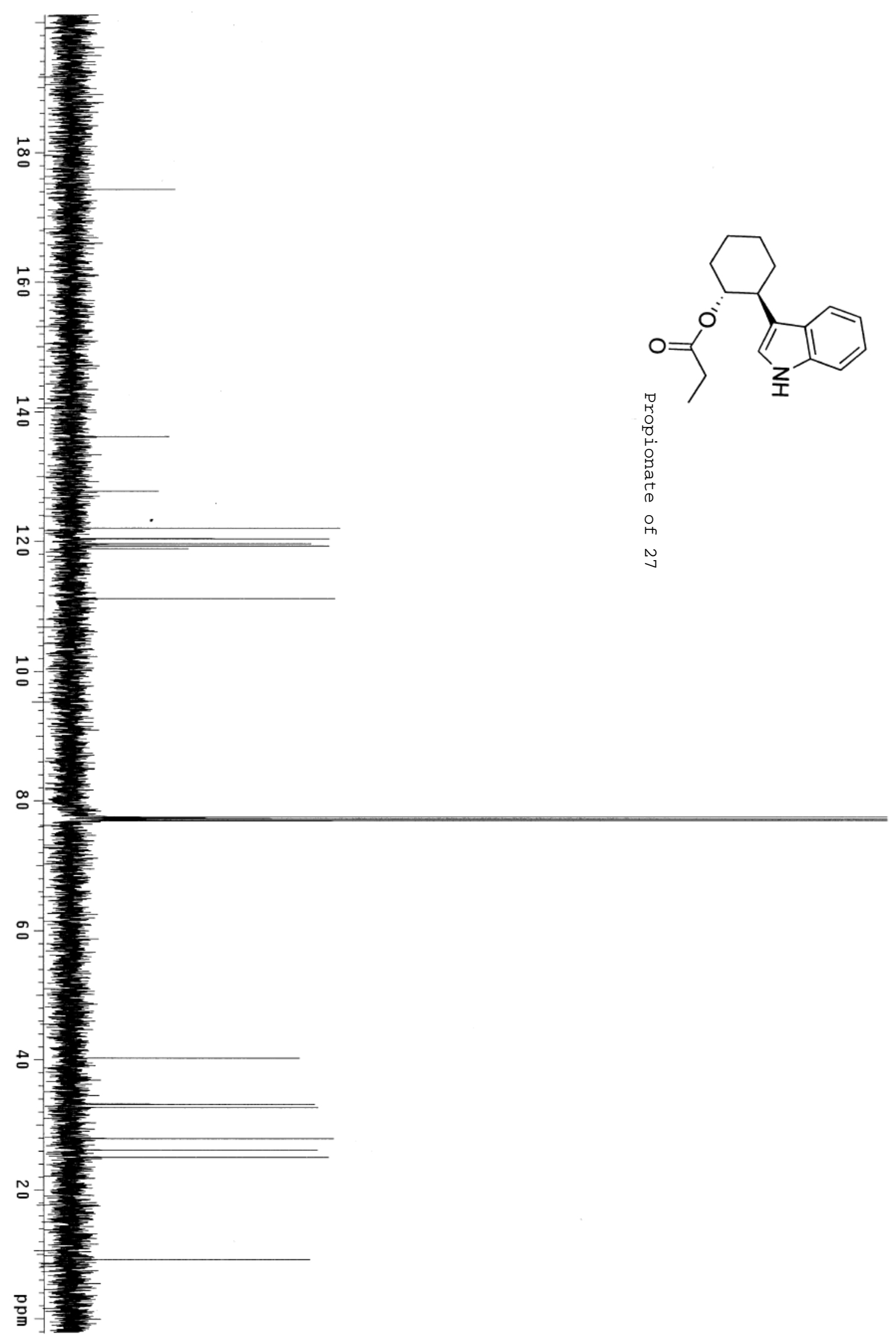

S-30 


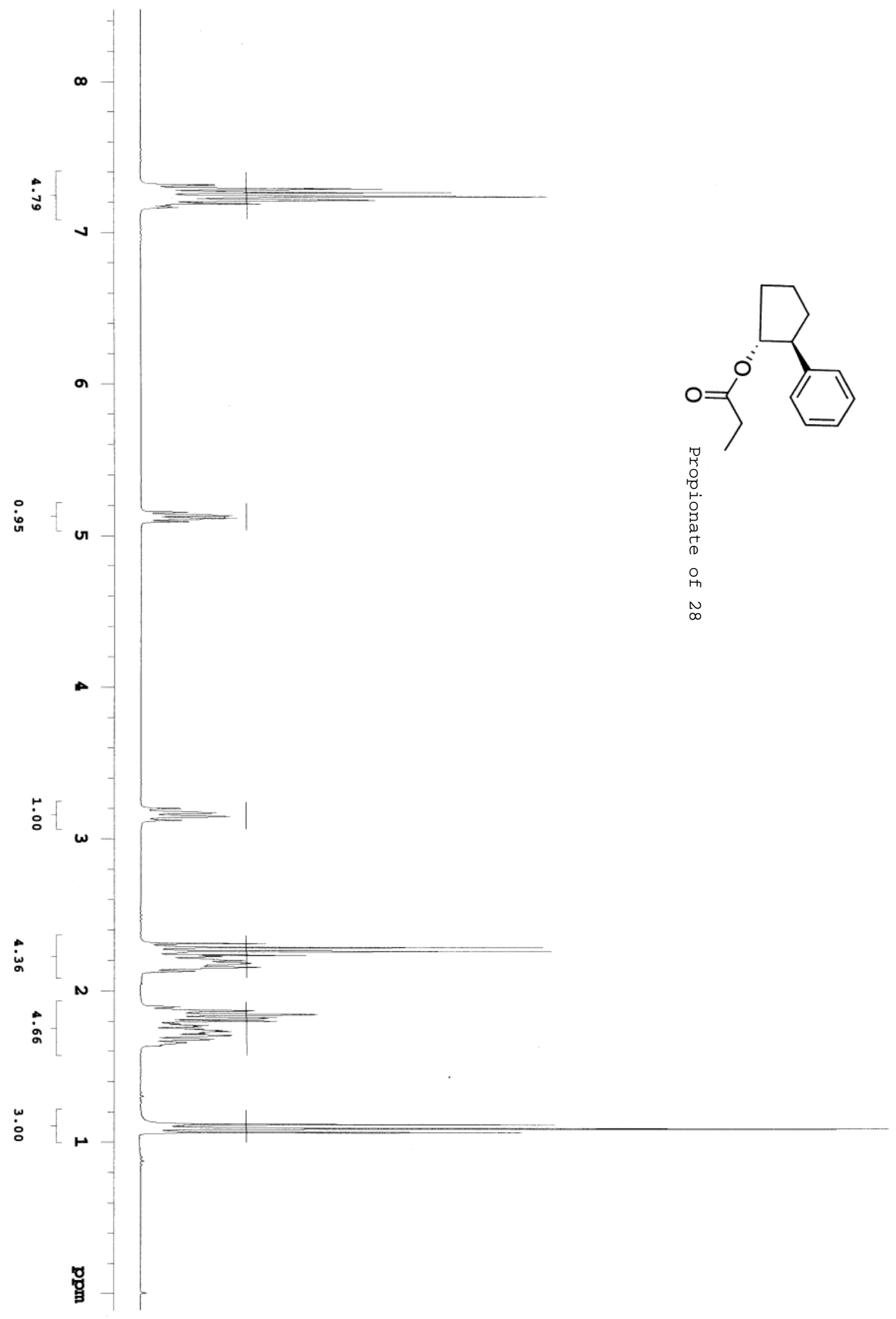

S-31 

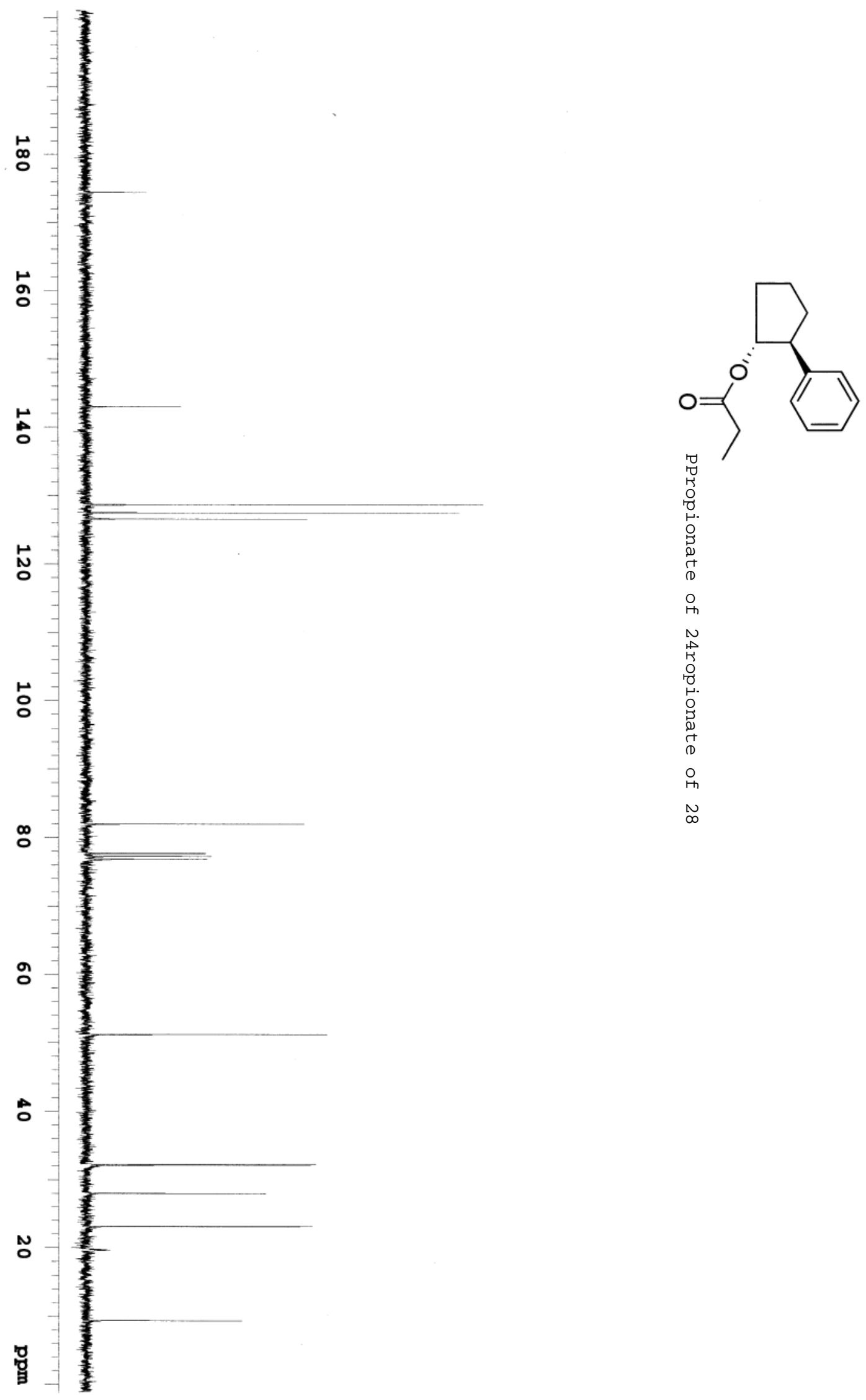

S-32 


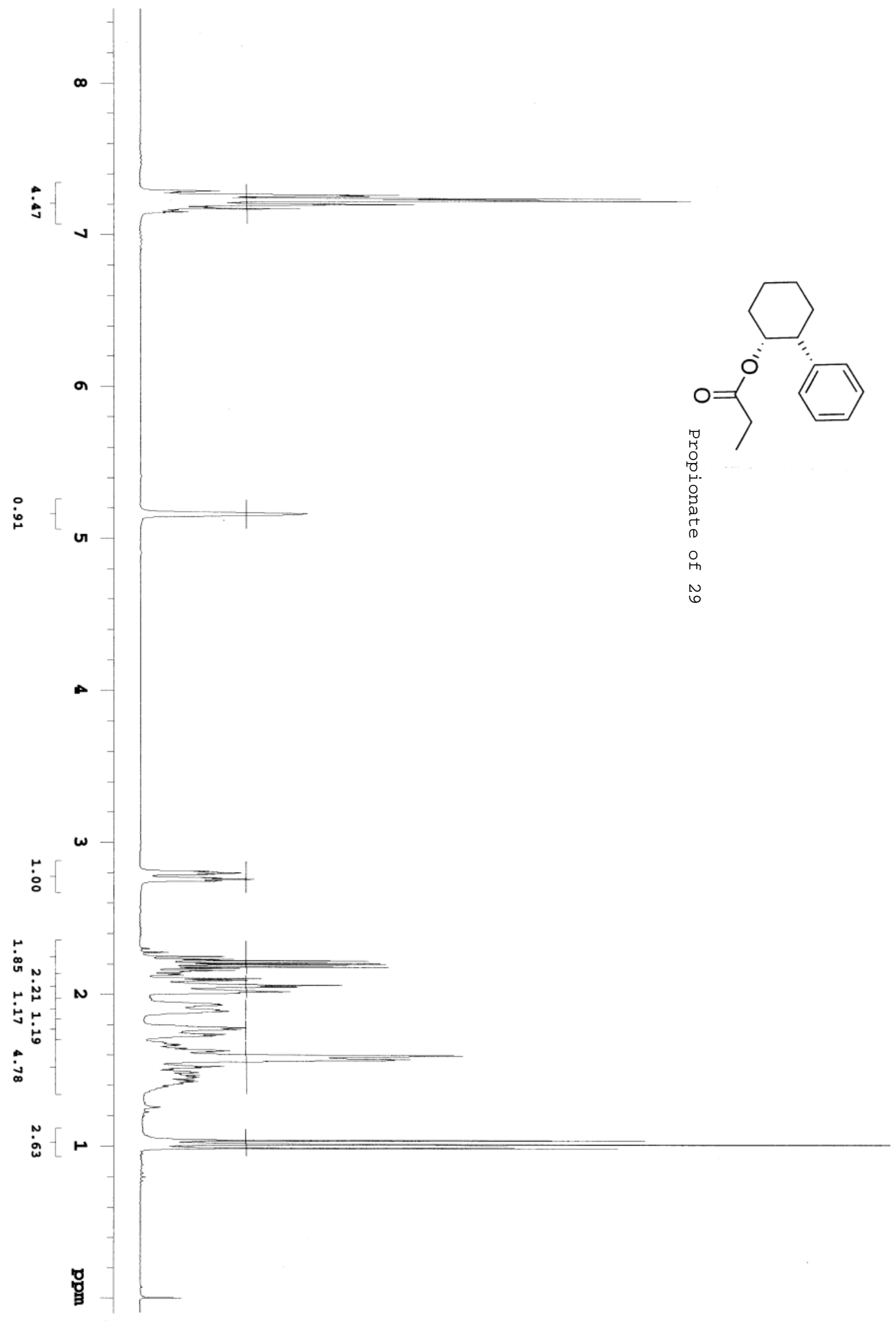

S-33 


$$
f
$$




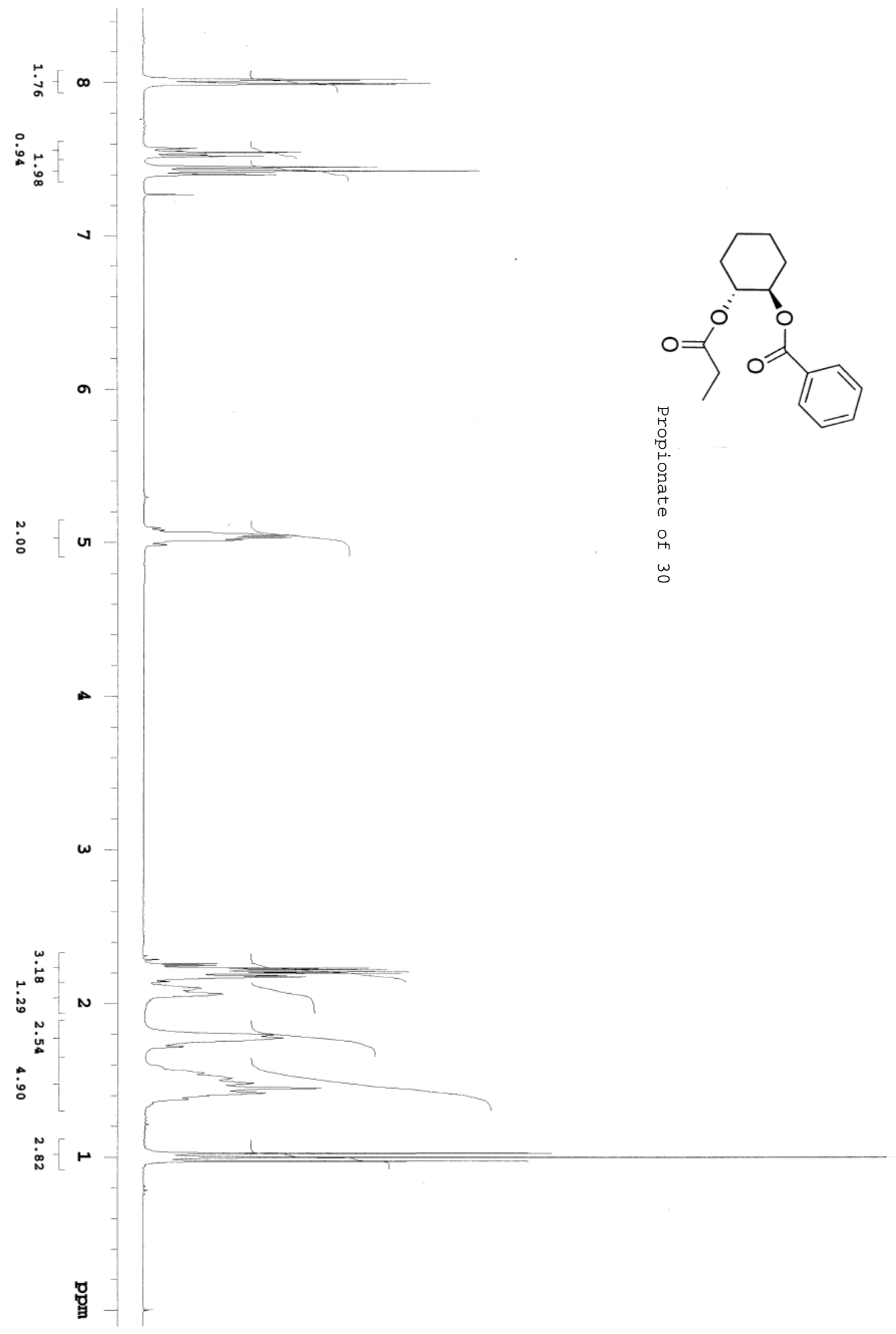

S-35 


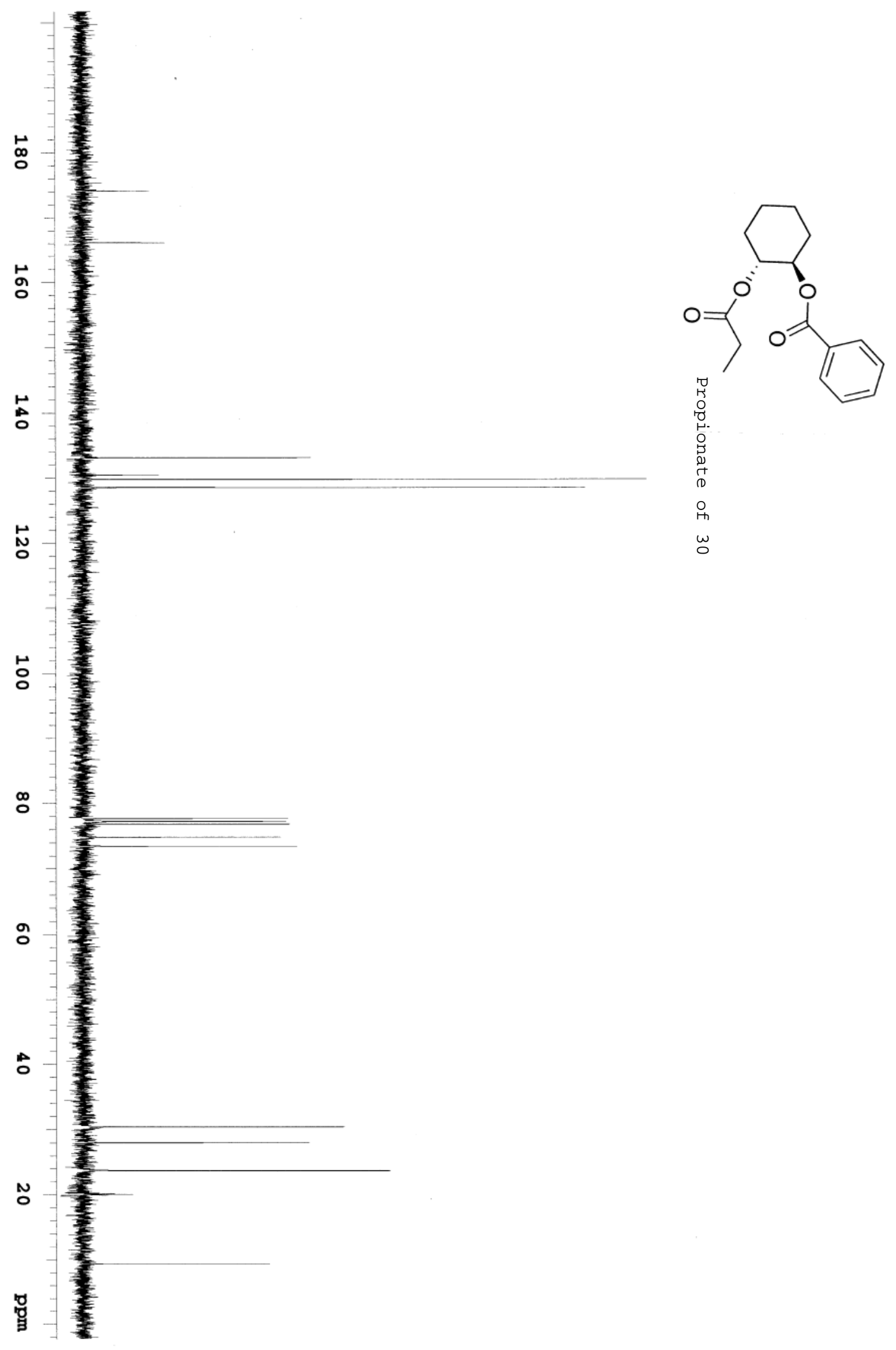

S-36 


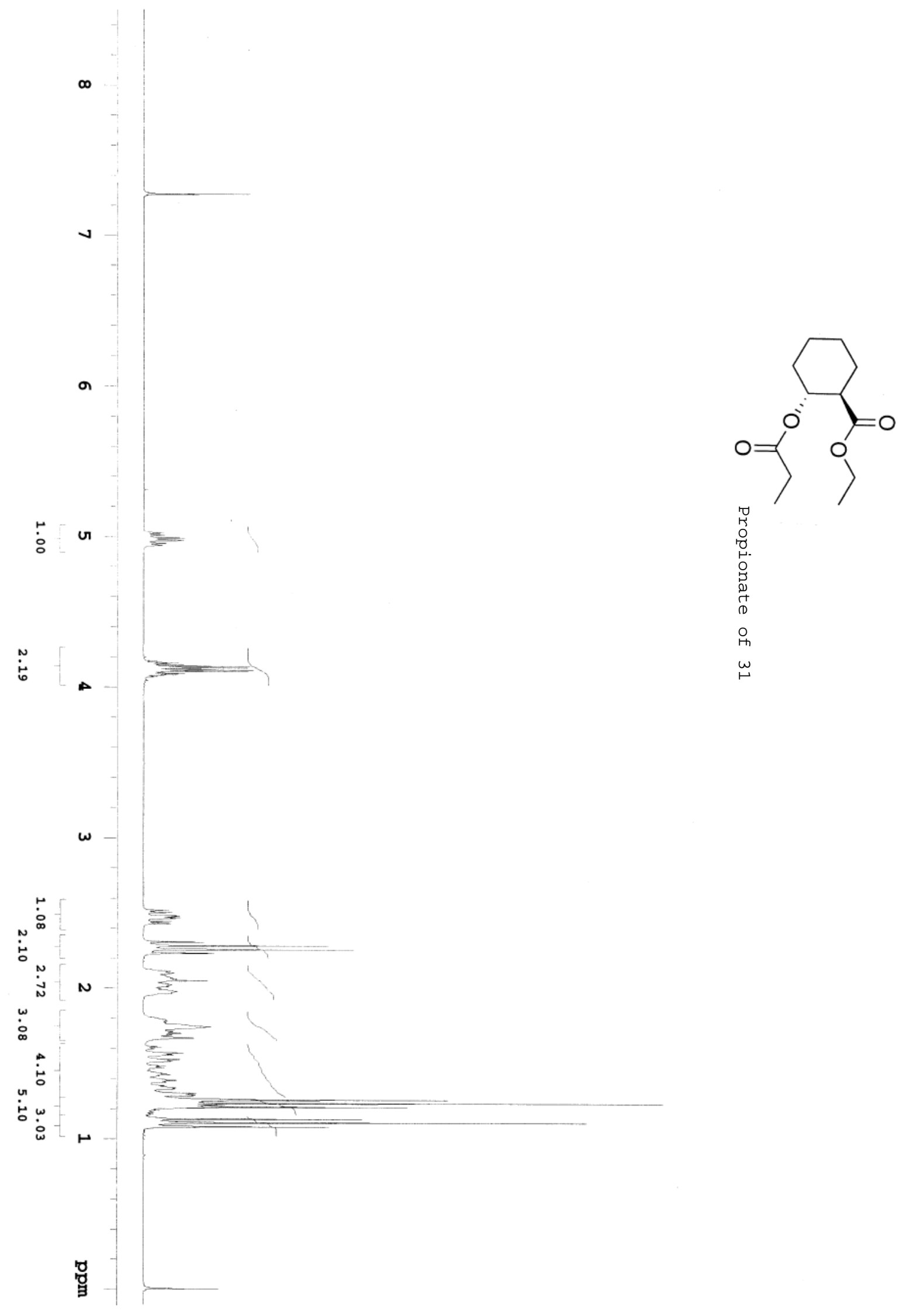



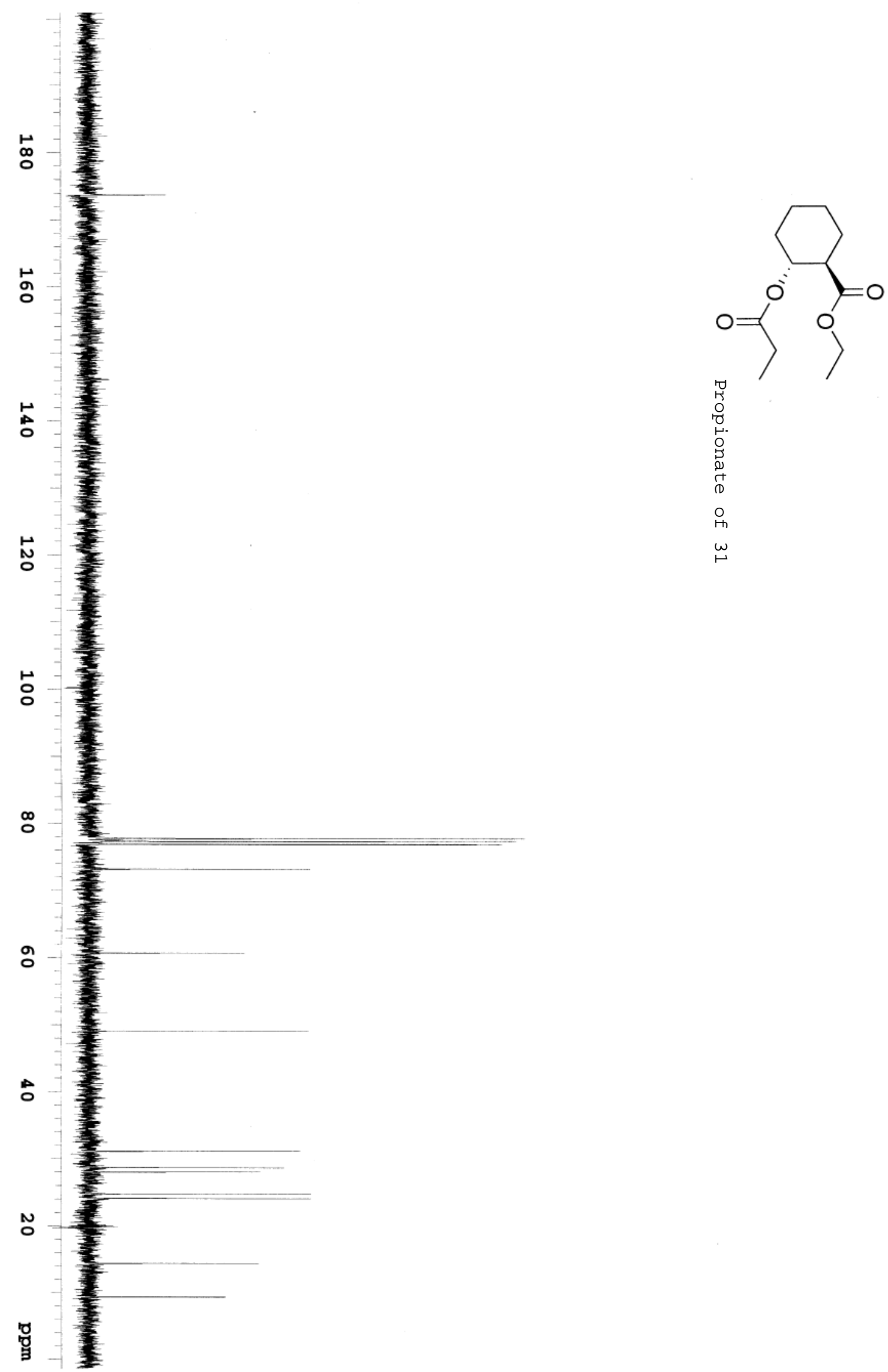

S-38 


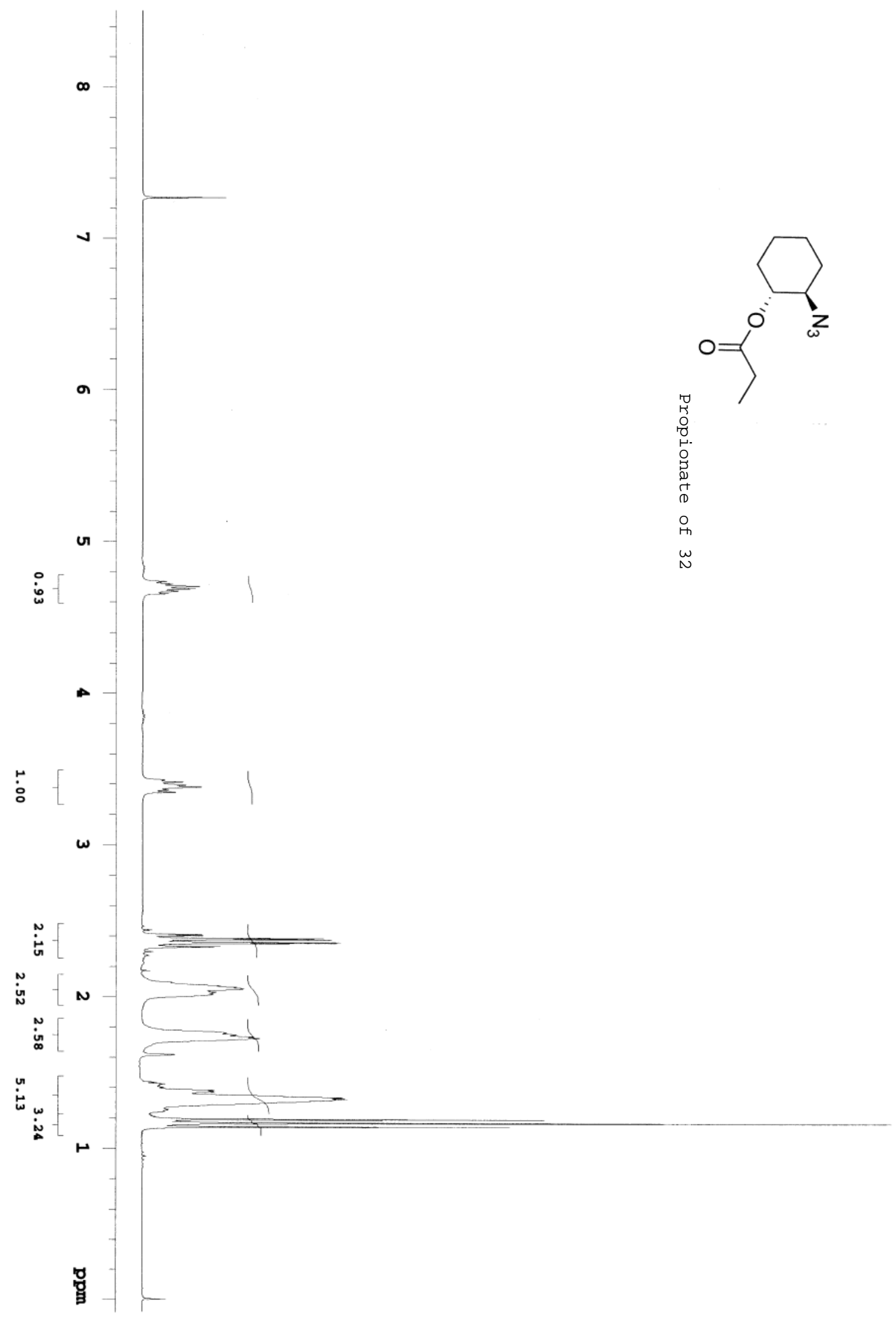



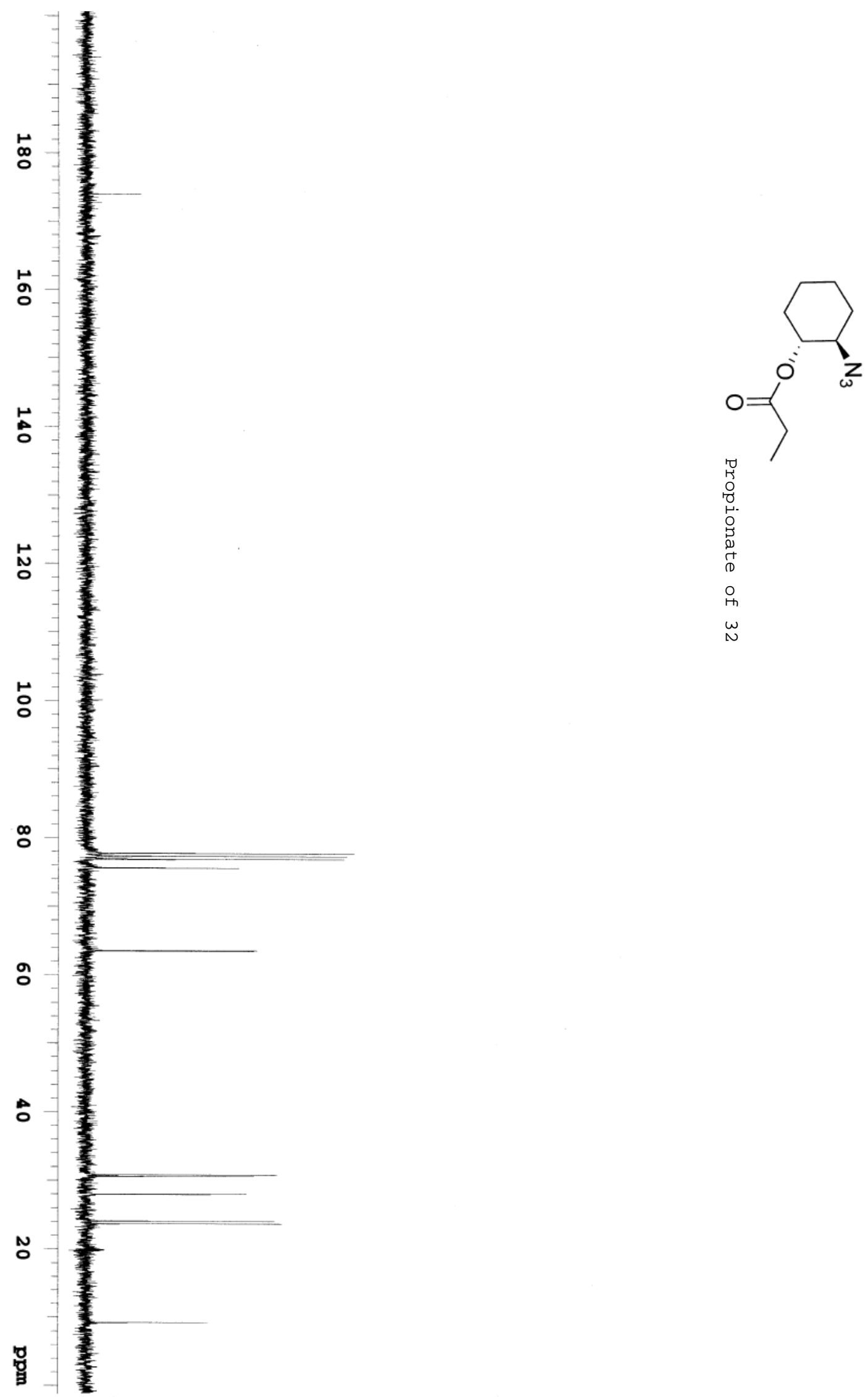